\title{
Physiological and pathophysiological homeostasis of astroglial channel proteins by
}

\section{Nedd4-2}

Bekir Altas ${ }^{1,2, *}$, Anes $\mathrm{Ju}^{1,3,13}$, Hugo Cruces Solís ${ }^{1,2,4,13}$, Samir Karaca ${ }^{2,5,13}$, Jan Winchenbach $^{3,4}$, Öyküm Kaplan ${ }^{1,3}$, Manuela Schwark ${ }^{1}$, Georg L. Wieser ${ }^{6}$, Viduth K. Chaugule $^{7}$, Irina Majoul ${ }^{8}$, Mohamed A. Hassan ${ }^{1}$, Rashi Goel ${ }^{1}$, Sonja M. Wojcik ${ }^{1}$, Andrea Pichler $^{7}$, Miso Mitkovski ${ }^{6}$, Livia de $\mathrm{Hoz}^{4}$, Henning Urlaub ${ }^{5,9}$, Olaf Jahn ${ }^{10}$, Gesine Saher ${ }^{4}$, JeongSeop Rhee ${ }^{1}$, and Hiroshi Kawabe ${ }^{1,11,12, *}$

${ }^{1}$ Department of Molecular Neurobiology, Max Planck Institute of Experimental Medicine, Hermann-Rein-Str. 3D, 37075, Göttingen, Germany

${ }^{2}$ International Max Planck Research School and the Göttingen Graduate School for Neurosciences, Biophysics and Molecular Biosciences, Grisebachstr. 5, 37077 Göttingen, Germany

${ }^{3}$ University of Göttingen, the Göttingen Graduate School for Neurosciences, Biophysics, and Molecular Biosciences, PhD program Systems Neuroscience, Kellnerweg 4, 37077 Göttingen, Germany

${ }^{4}$ Department of Neurogenetics, Max Planck Institute of Experimental Medicine, HermannRein-Str. 3D, 37075, Göttingen, Germany

${ }^{5}$ Bioanalytical Mass Spectrometry Group, Max Planck Institute for Biophysical Chemistry, Am Fassberg 11, D-37077, Göttingen, Germany

${ }^{6}$ Light Microscopy Facility, Max Planck Institute of Experimental Medicine, Hermann-ReinStr. 3D, 37075, Göttingen, Germany

${ }^{7}$ Department of Epigenetics, Max Planck Institute of Immunobiology and Epigenetics, Stübeweg 51, 79108, Freiburg, Germany 
${ }^{8}$ Institute of Biology, Center for Structural and Cell Biology in Medicine, University of Lübeck, Ratzeburger Allee 160, 23562, Lübeck, Germany

${ }^{9}$ Bioanalytical Group, Institute for Clinical Chemistry, University Medical Center Göttingen, Robert Koch Str. 40, 37075 Göttingen, Germany

${ }^{10}$ Proteomics Group, Max Planck Institute of Experimental Medicine, Hermann-Rein-Str. 3, 37075, Göttingen, Germany

${ }^{11}$ Division of Pathogenetic Signaling, Department of Biochemistry and Molecular Biology, Kobe University Graduate School of Medicine, 1-5-6 Minatojima-minamimachi, Chuo-ku, Kobe, 650-0047, Japan

${ }^{12}$ Department of Gerontology, Laboratory of Molecular Life Science, Institute of Biomedical Research and Innovation, Foundation for Biomedical Research and Innovation at Kobe, 2-2 Minatojima-Minamimachi Chuo-ku, Kobe 650-0047, Japan

${ }^{13}$ These authors contributed equally

* Corresponding authors; Bekir Altas (altas@em.mpg.de) and Hiroshi Kawabe (kawabe@em.mpg.de).

Mailing address for both; Department of Molecular Neurobiology, Max Planck Institute of Experimental Medicine, Hermann-Rein-Str. 3D-37075, Goettingen, Germany.

ORCID for Hiroshi Kawbae; https://orcid.org/0000-0001-5650-8696

\section{CONDENSED TITLE:}

Ubiquitination of Astrocytic Channels by Nedd4-2 


\section{SUMMARY:}

Ubiquitination is a key regulatory machinery for protein expression. Our present work provides evidence that astrocytic ion channel proteostasis coordinated by an E3 ubiquitin ligase Nedd4-2 is of particular importance for the maintenance of neuronal network activity. 


\begin{abstract}
Nedd4-2 is an E3 ubiquitin ligase, missense mutation of which is related to familial epilepsy, indicating its critical role in regulating neuronal network activity. However, Nedd4-2 substrates involved in neuronal network function have yet to be identified. Using mouse lines lacking Nedd4-1 and Nedd4-2, we identified astrocytic channel proteins inwardly rectifying $\mathrm{K}^{+}$channel 4.1 (Kir4.1) and Connexin43 as Nedd4-2 substrates. We found that the expression of Kir4.1 and Connexin43 is increased upon conditional deletion of Nedd4-2 in astrocytes, leading to an elevation of astrocytic membrane ion permeability and gap junction activity, with a consequent reduction of $\gamma$-oscillatory neuronal network activity. Interestingly, our biochemical data demonstrate that missense mutations found in familial epileptic patients produce gain-of-function of Nedd4-2 gene product. Our data reveal a process of coordinated astrocytic ion channel proteostasis that controls astrocyte function and astrocyte-dependent neuronal network activity, and elucidate a potential mechanism by which aberrant Nedd4-2 function leads to epilepsy.
\end{abstract}




\section{INTRODUCTION}

Neuronal network activity depends critically on the properties of the nerve cells involved e.g. synaptic connectivity, synapse strength, and excitation-inhibition balance. In addition, glia cells play important roles as modulators of neuronal and circuit function. For instance, gliotransmitters released from astrocytes control neuronal functions by binding to NMDA receptors (Araque et al., 1998; Clasadonte et al., 2013), the release of S100 $\beta$ induces $\gamma$ oscillations (Sakatani et al., 2008), and astrocytes act as important regulators of ion homeostasis in the brain via astrocytic ion channels, ion pumps, and gap junctions. All of them play roles in ion and solute exchange with the extracellular space and between adhering astrocytes (Chever et al., 2010; Haglund and Schwartzkroin, 1990; Takumi et al., 1995; Wallraff et al., 2006), thus acutely regulating the extracellular environment that determines neuronal excitability.

The most prominent $\mathrm{K}^{+}$-permeable astrocytic channel is the inwardly rectifying $\mathrm{K}^{+}$channel 4.1 (Kir4.1), which plays a key role in the clearance of excess extracellular $\mathrm{K}^{+}$. Its conditional knock-out $(\mathrm{KO})$ in mice causes a reduction of electrophysiologically measured whole-cell ion currents in astrocytes, and $\mathrm{KO}$ animals show pronounced body tremor, ataxia, and tonicclonic seizures (Djukic et al., 2007). Similar symptoms are seen in patients with SeSAME- or EAST-syndrome, who carry frame-shift or missense mutations in the coding sequence of KCNJ10, the human orthologue of Kir4.1 gene (Bockenhauer et al., 2009; Scholl et al., 2009). Extracellular $\mathrm{K}^{+}$taken up by astrocytes is quickly dissipated within a gap-junction-connected astrocytic network. The corresponding astrocytic gap junctions are mainly composed of Connexin43 (Cx43) and Connexin30 (Cx30) (Wallraff et al., 2006). In mice lacking Cx30 and $\mathrm{Cx} 43$, spontaneous epileptiform field potentials are seen in the CA1 region of hippocampus (Wallraff et al., 2006), demonstrating that astrocytic gap-junction-coupling is required for the dampening of neuronal network activity (Pannasch et al., 2011). 
The cell surface expression and turnover of ion channels is regulated by protein ubiquitination (Kimura et al., 2011; Staub and Rotin, 2006; Zhou et al., 2007). It is mediated by the successive action of ubiquitin (Ubi) activating enzymes (E1), Ubi conjugating enzymes (E2), and Ubi ligases (E3), of which the E3s define substrate specificity (Hershko and Ciechanover, 1998). Ubi itself has seven lysine residues that can act as Ubi acceptors, so that various types of polyUbi chains can be formed. The most widely known K48-linked polyUbi chains target substrate proteins to proteasomal degradation, while plasma membrane proteins with K63-linked polyUbi chains are endocytosed and lysosomally degraded (Piper et al., 2014). Neuronal precursor expressed developmentally downregulated 4 (Nedd4) family E3s have been implicated in monoubiquitination and K63-linked polyUbi chain formation (Kawabe et al., 2010; Maspero et al., 2013; Woelk et al., 2006). They belong to the homologous to the E6AP carboxyl terminus (HECT)-type E3 superfamily. Among the Nedd4 family E3s, Nedd4-2 (N4-2) is of particular interest because missense mutations of evolutionarily conserved residues (S233L, E271A, H515P) were found in patients with familial photosensitive epilepsy (Dibbens et al., 2007; Vanli-Yavuz et al., 2015) and several other de novo mutations in N4-2 were reported in epilepsy patients (Allen et al., 2013), indicating that N4-2 is a critical regulator of neuronal network activity.

In the present study, we identified Kir4.1 and Cx43 as most prominent substrates of N4-2 in a quantitative mass spectrometric screen. We found that N4-2-deficient astrocytes exhibit elevated levels of Kir4.1 and Cx43, which cause increased plasma membrane ion permeability and gap junction coupling. Reflecting these cellular changes, astrocyte-specific N4-2 KO mice show severely altered neuronal network synchronicity as indicated by a strong reduction of the power of $\gamma$-oscillatory activity in the brain. This deficiency is restored by the application of pharmacological blockers of Kir4.1 or $\mathrm{Cx} 43$. Our data unravel a novel regulatory principle by which a single E3 ligase N4-2 coordinately controls the surface expression of two ion channels critical for fundamental aspects of astrocyte function, i.e. the 
plasma membrane ion permeability and gap junction network connectivity, which, in turn, are key determinants of neuronal network synchronicity. Additionally, we determine that pathological N4-2 point mutations found in familial photosensitive epilepsy patients are gainof-function-mutations, indicating that inappropriate enhancement of N4-2-dependent ubiquitination and degradation of Kir4.1 and $\mathrm{Cx} 43$ could be a potential cause of familial epilepsy. 


\section{RESULTS}

\section{N4-2 is a Critical Regulator of Neuronal Network Synchronicity}

Missense mutations of evolutionarily conserved residues of N4-2 (S233L, E271A, H515P) were found in patients with familial photosensitive epilepsy (Dibbens et al., 2007; VanliYavuz et al., 2015). Given that enhanced $\gamma$-band oscillatory activities could be one of potential triggers of photosensitive epilepsy (Parra et al., 2003; Perry et al., 2014), we studied the impact of loss of N4-2 alone or together with its closest isoform Nedd4-1 (N4-1) on $\gamma$ oscillations in hippocampal CA3 region of acute brain slices. $\gamma$-Oscillations were induced by $100 \mathrm{nM}$ kainate and recorded as local field potentials (LFPs). The average power of $\gamma$ oscillatory activity was significantly reduced, rather than enhanced, in brain-specific conditional N4-1/N4-2 KO mice $\left(N e d d 4-1^{\mathrm{f} / \mathrm{f}} ; N e d d 4-2^{\mathrm{f} / \mathrm{f}} ; E M X\right.$-Cre; N4-1/2 bDKO) (Gorski et al., 2002; Kawabe et al., 2010; Kimura et al., 2011) and in brain-specific N4-2 KO mice $\left(\right.$ Nedd4- $2^{\mathrm{f} / \mathrm{f}} ; E M X$-Cre; N4-2 bKO) as compared to controls (Figure 1). This phenotype was more pronounced in N4-1/2 bDKO than in N4-2 bKO, indicating that N4-2 plays a prominent role to maintain the neuronal network activity and that N4-2 loss is compensated by N4-1 partially.

\section{Gain of E3 Function by a Missense Mutation of N4-2 in Epileptic Patients}

Amino acid residues mutated in N4-2 of familial photosensitive epilepsy patients (S233L, E271A, H515P) are not located within the substrate-recognizing WW domains (Dibbens et al., 2007; Vanli-Yavuz et al., 2015). We thus hypothesized that these mutations might affect the intrinsic enzymatic activity of N4-2 rather than substrate binding. To test this hypothesis, we purified recombinant WT and two mutant (S233L and H515P) N4-2 proteins and analyzed their enzymatic activities by in vitro ubiquitination assays with Ubi as substrate. In these experiments, the reduction of monomeric Ubi (i.e. free Ubi) and the formation of polyUbi chains serve as readouts for N4-2 enzyme activity (Figures 2A and 2B). The 
depletion of monomeric Ubi and formation of polyUbi chains in this assay was significantly enhanced in mutant N4-2 within the linear range of the assay time course (Figures 2C-2F). These results indicate that the individual S233L and H515P mutations cause a gain-offunction of N4-2 E3 activity, which might result in increased ubiquitination levels and reduced expression of substrates in patients' brains and in increased power of $\gamma$-oscillations as reported in photosensitive epileptic patients (Perry et al., 2014).

\section{A Screen for N4-1 and N4-2 Substrates in the Brain Identifies Kir4.1 and Cx43}

N4-1 and N4-2 generate K63-linked polyUbi chains (Maspero et al., 2013), which are involved in the endocytosis and lysosomal degradation of transmembrane proteins (Acconcia et al., 2009). In contrast to a previous study (Zhu et al., 2017), the levels of the glutamate receptor subunit GluA1 were not changed in N4-1/2 bDKOs (Figures 3A-3C), indicating that the reduced power of $\gamma$-oscillations in N4-1/2 bDKOs is not caused by aberrant AMPA receptor activity. After determining that cortex expresses N4-1 and N4-2 at the levels comparable to those in hippocampus (data not shown), we purified synaptic plasma membrane fractions (SM) from N4-1/2 bDKO and N4-1/2 CTL cortices (Figure 3D) (Mizoguchi et al., 1989) and compared protein profiles using isobaric tags for relative and absolute quantitation (iTRAQ) (Ross et al., 2004; Schmidt et al., 2013). Intriguingly, two astrocytic membrane proteins, the $\mathrm{K}^{+}$channel Kir4.1 and the gap junction alpha 1 protein (Gja1 or $\mathrm{Cx} 43$ ) were found as the two most upregulated proteins in N4-1/2 bDKO samples (Figure 3E, Table S1). The results obtained by proteomic screening were validated by quantitative Western blotting for Kir4.1 and Cx43 in N4-1/2 bDKO and N4-1/2 CTL samples (Figures 3F-3H). Levels of EAAT2, which has a subcellular distribution akin to Kir4.1 and Cx43, were not changed in N4-1/2 bDKO samples (Figures 3D, 3I, and 3J), demonstrating that the upregulation of Kir4.1 and $\mathrm{Cx} 43$ in N4-1/2 bDKO samples is not due to an increase in astrocyte numbers in N4-1/2 bDKO cortex. The levels of Kir4.1 and Cx43 mRNAs were 
not different between N4-1/2 bDKO and control samples, indicating that the modulatory effects of the N4-1/2 bDKO on Kir4.1 and $\mathrm{Cx} 43$ levels arise post-transcriptionally (Figures $3 \mathrm{~K}$ and $3 \mathrm{~L})$.

\section{N4-2 Plays Dominant Roles for the Regulation of Kir4.1 and Cx43}

We found that the levels of Kir4.1 and Cx43 in brain-specific N4-1 single KOs $\left(N 4-1^{\mathrm{f} / \mathrm{f}} ; E M X\right.$ Cre; N4-1 bKO) were unchanged as compared to littermate controls (N4-1 ${ }^{\mathrm{f} / \mathrm{f}}$; N4-1 CTL), while brain-specific N4-2 single KOs $\left(N 4-2^{\mathrm{f} / \mathrm{f}} ; E M X\right.$-Cre; N4-2 bKO) showed significant increases in Kir4.1 and Cx43 as compared to littermate controls (N4-2 ${ }^{\mathrm{f} / \mathrm{f}}$; N4-2 CTL) (Figures 3M-3R). The fold changes of Kir4.1 and Cx43 levels in N4-2 bKOs are smaller than in N41/2 bDKOs (compare Figures 3Q and 3R with Figures 3G and 3H), indicating that N4-2 is the dominant E3 for Kir4.1 and Cx43 and that N4-1 compensates the loss of N4-2 partially.

\section{Ubiquitination of Kir4.1 and Cx43 by N4-1 and N4-2}

To test if N4-1 and N4-2 are able to ubiquitinate Kir4.1 and Cx43, we employed recombinant cell-based ubiquitination assays. Ubiquitination of HA-tagged Kir4.1 (Kir4.1-HA) or Cx43 (Cx43-HA) by EGFP-tagged Nedd4 E3s (EGFP-N4-1 and EGFP-N4-2) was readily detected by anti-Ubi antibody labeling (third panels in Figures 4A and 4B). Interestingly, Ubi signals from Kir4.1-HA and Cx43-HA samples were more prominent in EGFP-N4-2-overexpressing cells than in EGFP-N4-1-overexpressing cells, although EGFP-N4-1 was expressed more than EGFP-N4-2 (top panels in Figures 4A and 4B), indicating that N4-2 is a more efficient E3 for the ubiquitination of Kir4.1 and Cx43 than N4-1.

Although mammalian Nedd4 subfamily E3s form K63-linked polyUbi chains (K63-Ubchains) preferentially (Maspero et al., 2013), their yeast orthologue Rsp5 has been demonstrated to conjugate K48-linked polyUbi chains (K48-Ub-chains) to substrates (Fang et al., 2014). To investigate whether Kir4.1 and Cx43 are conjugated with K63- or K48-Ub- 
chains, cell-based ubiquitination samples were immunoblotted with antibodies specific for K48- and K63-Ub-chains (bottom two panels in Figures 4A and 4B). Signals from Kir4.1HA detected by both antibodies were pronounced in EGFP-N4-2-dependent manner, indicating that N4-2 conjugates K48- and K63-Ub-chains to Kir4.1. Cx43-HA immunoprecipitated from HEK293FT cells showed a robust signal from the anti-K48-Ubchain antibody in the absence of recombinant N4-1 or N4-2 (the third lane in the fourth panel in Figure 4B). This is probably caused for the clearance of excess Cx43-HA by endoplasmic reticulum-associated degradation (ERAD) system, where K48-Ub-chains play a crucial role. The signal from K48-Ub-chains was diminished upon overexpression of N4-1 or N4-2 (the first and second lanes in the fourth panel in Figure 4B) whereas K63-Ub-chains conjugated with Cx43-HA were increased (the bottom panel in Figure 4B). We interpret this data as compensatory homeostatic regulation of $\mathrm{Cx} 43-\mathrm{HA}$ by K63-ubiquitination-dependent lysosomal degradation. Western blotting in the bottom panel of Figure 4B highlights that N42 is the dominant E3 for Cx43 whereas N4-1 has a ligase activity to some extent. Accordingly, ubiquitination of Kir4.1 and Cx43 was clearly reduced in primary astrocytes derived from N4-1/2 bDKO as compared to N4-1/2 CTLs, indicating that endogenous N4-1 and N4-2 plays crucial roles in the ubiquitination of Kir4.1 and Cx43 in astrocytes (Figures 4C and 4D). Small fractions of Kir4.1-HA and Cx43-HA remained ubiquitinated in N4-1/2 DKO cells (third lanes in Figure 4C and 4D) probably by other types of E3s involved in ERAD or lysosomal degradation (e.g. Hrd-1 and c-Cbl).

Using recombinant full-length N4-1, N4-2, and fragments of N4-2 (Figure 4E), we compared affinities of N4-1 and N4-2 for Kir4.1-HA and Cx43-HA and mapped relevant substrate binding regions. Figure $4 \mathrm{~F}$ shows that $\sim 2$-fold more Kir4.1-HA and Cx43-HA bound to purified GST-tagged N4-2 than to GST-N4-1. This result together with data in Figures 3M$3 \mathrm{R}, 4 \mathrm{~A}$, and $4 \mathrm{~B}$ supports the notion that N4-2 is the prominent ligase regulating the ubiquitination and expression levels of Kir4.1 and Cx43, while N4-1 plays a limited role. 
Mapping of the substrate-binding region of N4-2 demonstrated that the third and fourth WW domains of N4-2 are sufficient to bind to Kir4.1 and Cx43 (Figure 4F).

\section{Downregulation of Kir4.1 and Cx43 in Astrocytes Depends on N4-2 Ligase Activity}

Next, primary cortical astrocyte cultures were prepared from N4-1/2 bDKOs and N4-1/2 CTLs, and recombinant wild-type N4-2 (N4-2 WT) or a catalytically inactive point mutant of N4-2 (N4-2 C/S) protein was expressed to study levels of Kir4.1 and Cx43 (Figure 4G). The reintroduction of recombinant N4-2 WT in N4-1/2 bDKO astrocytes readily restored Kir4.1 and $\mathrm{Cx} 43$ protein abundance to control levels, while expression of N4-2 C/S failed, demonstrating that the levels of Kir4.1 and $\mathrm{Cx} 43$ in astrocytes are dependent on the enzymatic activity of N4-2.

\section{Blockade of the Endo-Lysosomal Pathway in Astrocytes Phenocopies N4-1/2 bDKO}

While K48-linked polyUbi chains are directly recognized by the proteasome and play crucial roles in ERAD, K63-linked chains are crucial for the transport toward the endo-lysosomal pathway. To study whether N4-2 promotes the degradation of Kir4.1 and Cx43 via the endolysosomal pathway, clathrin-mediated endocytosis was blocked with dynasore, a specific blocker of dynamin (Macia et al., 2006) in primary astrocyte cultures (Figure 4H). Dynasore treatment increased the levels of Kir4.1 and Cx43 in N4-1/2 CTL astrocytes to the levels seen in N4-1/2 bDKO astrocytes and the effects of dynasore were more pronounced in N4-1/2 CTL astrocytes than in N4-1/2 bDKO astrocytes. This indicates that dynasore phenocopies the genetic ablation of N4-1 and N4-2, and that endogenous N4-1 and N4-2 are required for the endo-lysosomal degradation of Kir4.1 and Cx43.

Increased Whole-Cell Currents and Reduced Membrane Resistance in N4-2 KO Astrocytes 
In view of the increased steady-state levels of Kir4.1 in N4-2 bKO mice, we measured wholecell currents and membrane resistance of cortical astrocytes embedded in acute cortical brain slices. Astrocytes stained with sulforhodamine 101 (Nimmerjahn et al., 2004) were wholecell voltage clamped and typical whole-cell passive currents were recorded with $10 \mathrm{mV}$ voltage steps from $-120 \mathrm{mV}$ to $+40 \mathrm{mV}$ (Figure 5). The whole-cell inward currents of astrocytic plasma membranes were increased (Figures 5A, 5B, 5D, and 5E) and their membrane resistance was reduced (Figure 5F) in the absence of N4-2. These electrophysiological defects were efficiently restored by the application of Kir4.1 inhibitor fluoxetine (Figures 5C-5F), depicting that the augmented Kir4.1 level in N4-2 bKO astrocytes results in an increase in membrane ion permeability.

\section{Increased Astrocyte Coupling upon Loss of N4-1 and N4-2}

Next, astrocyte couplings of primary cultured N4-1/2 bDKO astrocytes, but not N4-2 bKO cells, were studied, given the potential E3 activity of N4-1 towards Cx43 (the bottom panel in Figure 4B). Cultured astrocytes were prepared from the cortex but not hippocampus because of homogenous expression patterns of $\mathrm{Cx} 43$ and N4-2 throughout the cortex and hippocampus (Allen Brain Atlas; http://mouse.brain-map.org/). Primary cortical astrocyte cultures were loaded with the membrane-permeable acetomethoxy (AM) derivative of calcein (Abbaci et al., 2007). The AM ester group is cleaved off by intracellular esterases, resulting in a membrane-impermeable form of the fluorophore, calcein. Calcein is a small compound with a molecular weight of $\sim 600 \mathrm{Da}$ and can diffuse through gap junctions. To estimate intercellular coupling, calcein in single cells was photobleached and fluorescence recovery after photobleaching (FRAP) was recorded in bleached cells by time-lapse confocal microscopy. Relative FRAP in N4-1/2 bDKO astrocytes was significantly faster than that in N4-1/2 CTL astrocytes (Figure 6), indicating that the elevation of Cx43 leads to an increased syncytial connectivity in the astrocyte networks lacking N4-1 and N4-2. 


\section{Reduced $\gamma$-Oscillatory Activity upon Loss of N4-2 from Astrocytes}

To test whether altered $\gamma$-oscillatory activity shown in Figure 1 is the direct consequence of N4-2 loss in astrocytes, we crossed N4-2 floxed mice with tamoxifen-inducible astrocytespecific Cre driver mice (Aldh1L1-CreERT2) (Winchenbach et al., 2016). Using our tamoxifen induction protocol, the specificity and efficiency of Cre recombination were confirmed by studying the expression profile of a Cre-dependent reporter protein tdTomato (tdTom) in Aldh1L1-CreERT2;Rosa26-tdTom mice (Figures 7A-7C)(Madisen et al., 2010). The astrocyte-specific N4-2 KO mouse (N4-2 ${ }^{\mathrm{f} / \mathrm{f}}$;Aldh1L1-CreERT2, N4-2 AstKO) showed a significant reduction of N4-2 protein expression and increases in Kir4.1 and Cx43 (Figures 7D-7G), as well as Kir4.1-dependent increase in currents of astrocyte plasma membrane (Figures $7 \mathrm{H}-7 \mathrm{M})$. The number of parvalbumin (PV)-positive interneurons, which are also critically involved in hippocampal CA3 $\gamma$-oscillations (Cardin et al., 2009; Gloveli et al., 2005), was found to be unchanged in the CA3 region of N4-2 AstKOs (Figures 7N-7P). As observed in N4-2 bKO (Figure 1E-1H), the average power of $\gamma$-oscillatory activity was reduced in N4-2 AstKO as compared to N4-2 CTL significantly (Figure 8). The power of $\gamma$ oscillations was also reduced in the hippocampal CA3 region in anesthetized N4-2 AstKO mice in vivo (Figure 9). These findings show that the loss of N4-2 specifically in astrocytes leads to decreased $\gamma$-oscillations in the hippocampus without influencing the number of PVpositive interneurons.

To investigate direct involvements of increases in Cx43 and Kir4.1 levels in the reduction of $\gamma$-oscillatory activity in N4-2 AstKOs, $\gamma$-oscillatory activity was recorded from CA3 hippocampal regions of acute brain slices in the presence of fluoxetine or GAP26, a blocker of Cx43 hemichannels and gap junctions (Chaytor et al., 1997). Both, fluoxetine (Figure 8C) and GAP26 (Figure 8H) occluded the effect of N4-2 loss on $\gamma$-oscillations, without influencing the oscillation frequency (Figures 8D, 8E, 8I, and 8J). We concluded that the N4- 
2-mediated downregulation of Kir4.1 and $\mathrm{Cx} 43$ in astrocytes is required for the maintenance of $\gamma$-band oscillatory activity in the CA3 region of the hippocampus (Figure 10). 


\section{DISCUSSION}

The present study (i) describes a novel cell biological mechanism by which a single E3 ligase, N4-2, coordinately ubiquitinates two key astrocytic ion channel proteins, Kir4.1 and Cx43, and thereby controls their surface expression to regulate astrocyte membrane $\mathrm{K}^{+}$conductance and gap junction coupling (Figures 3-6), and (ii) shows that this N4-2-dependent control of Kir4.1-mediated and Cx43-mediated astrocyte functions is a key determinant of synchronous nerve cell activity in the hippocampus (Figures 1,7-9). Beyond the biological significance of our findings described above, our study provides a possible mechanistic explanation of the etiopathology of photosensitive epilepsy in patients with point mutations in N4-2 (Figures 2 and 10).

\section{Identification of N4-2 E3 Ligase Substrates in the Brain}

Our proteomic screening approach identified the two astrocytic proteins Kir4.1 and Cx43 as the most strongly upregulated protein species in N4-1/2 bDKO brain (Table S1, Figure 3E). Subsequent biochemical (Figures 3F-3H) and qRT-PCR analyses (Figures 3K and 3L) verified that Kir4.1 and Cx43 are bona fide substrates of N4-2. These data identify a new level of regulation of the major astrocytic $\mathrm{K}^{+}$channel Kir4.1 that operates posttranslationally, i.e downstream of previously characterized transcriptional regulation (Farmer et al., 2016; Kelley et al., 2018), to control astrocyte function. Disruption of such Kir4.1 regulations could be the bases of several neurological disorders, including amyotrophic lateral sclerosis (Kelley et al., 2018) and depression (Cui et al., 2018). A recent report shows that N4-2-dependent ubiquitination of Kir4.1 is relevant for the maintenance of $\mathrm{K}^{+}$conductance in the kidney although proteome change in kidney-specific N4-2 KO is not shown (Wang et al., 2018). The fold change of Kir4.1 in kidney specific N4-2 KO (1.9-fold increase in (Wang et al., 2018)) is clearly smaller than what we found in N4-2 AstKO (more than 3-fold increase in Figure 7F), indicating the cell-type-specific regulation of Kir4.1 by N4-2 in astrocytes. 


\section{Consequences of N4-2 loss on Astrocyte Function}

Most E3 ligases are characterized by a rather broad substrate spectrum, and many proteins can be ubiquitinated by multiple E3s. Nevertheless, the major consequences of loss-offunction of a specific E3 in a given cell type are often due to aberrant ubiquitination of only a few substrates, indicating a substantial cell-type specificity and selectivity of E3-substrate interactions. The epithelial $\mathrm{Na}^{+}$channel $\mathrm{ENaC}$, one of the most prominent and bestestablished substrates of mammalian Nedd4-family E3s (Staub et al., 1996), is most prominently ubiquitinated by N4-2 in vitro (Zhou et al., 2007). ENaC ubiquitination and its subsequent downregulation are of particular importance for normal membrane $\mathrm{Na}^{+}$ conductance in kidney epithelial cells. Liddle syndrome patients, who suffer from hypertension, have frame-shift or point mutations in the ENaC gene that disrupt ENaCbinding to N4-2 (Tamura et al., 1996), leading to a loss of ubiquitination and consequent upregulation of ENaC (Firsov et al., 1996). In rodents, loss of N4-2 is accompanied by phenotypes that mimic Liddle syndrome patients in terms of altered ENaC-ubiquitination, ion balance, and blood pressure, confirming the critical and rather specific role of N4-2 in the regulation of $\mathrm{ENaC}$ levels and function in the kidney (Minegishi et al., 2016; Ronzaud et al., 2013).

The present study demonstrates that a dominant function of N4-2 in the adult brain is to specifically and selectively ubiquitinate and downregulate Kir4.1 and Cx43 in astrocytes. Loss of N4-2 leads to specific elevation of Kir4.1 and Cx43 protein levels (Figures 3P-3R and $7 \mathrm{D}-7 \mathrm{G})$, causing increases in the membrane ion permeability and in gap junction connectivity of astrocytes (Figures 5, 6, and 7H-7M). Astrocytic $\mathrm{K}^{+}$uptake is mediated by three major processes, involving $\mathrm{Kir} 4.1, \mathrm{Na}^{+}-\mathrm{K}^{+}-\mathrm{Cl}^{-}$cotransporters, and $\mathrm{Na}^{+} / \mathrm{K}^{+}$-ATPase. Although substantial amounts of $\mathrm{K}^{+}$are released by neurons during their repolarization after action potential firing, $\left[\mathrm{K}^{+}\right]_{\mathrm{o}}$ never exceeds the approximate ceiling level of $\sim 12 \mathrm{mM}$, not 
even during prolonged neuronal network activity (Heinemann and Lux, 1977). This is thought to be at least partly due to the fact that locally elevated $\left[\mathrm{K}^{+}\right]_{\mathrm{o}}$ is removed via Kir4.1 channels on the plasma membrane of astrocytes (Higashi et al., 2001; Karwoski et al., 1989), followed by the dissipation of the consequently and transiently increased astrocytic $\mathrm{K}^{+}$levels within the syncytial astrocytic network via gap junctions (Wallraff et al., 2006). Our data show that N4-2-dependent ubiquitination limits the maintenance of Kir4.1 and $\mathrm{Cx} 43$ on the astrocytic plasma membrane and promotes their lysosomal degradation, so that spatial $\mathrm{K}^{+}$ buffering by astrocytes is increased in the absence of N4-2 at two distinct levels, i.e. at the astrocyte surface via increased Kir4.1 activity and at astrocytic gap junctions via increased Cx43 levels (Figure 10).

\section{Non-Cell-Autonomous Roles of N4-2 in Neuronal Network Synchronicity}

The decreased $\gamma$-oscillations seen in N4-2 AstKO are reverted to normal levels by fluoxetine (Figure 8). Fluoxetine is relatively selective for Kir4.1, with only minor effects on other Kir channels at the concentration used in our experiments $(30 \mu \mathrm{M})(\mathrm{Ohno}$ et al., 2007). It still could have acted as a serotonin uptake inhibitor in our experiments, causing increases in extracellular serotonin levels (Malagie et al., 1995). However, it is unlikely that increased extracellular serotonin levels restore the phenotypic change in $\gamma$-oscillations in the N4-2 AstKO, because bath-application of serotonin actually blocks kainate induced $\gamma$-oscillations in rat hippocampal slices (Wojtowicz et al., 2009). Thus, we conclude that the increased astrocytic Kir4.1 levels are a major cause for the reduced power of $\gamma$-oscillations in N4-2 AstKOs, and that the phenotype-reverting effect of fluoxetine is mediated by a blockade of the excess Kir4.1 channels. It was reported recently that oligodendrocyte-specific Kir4.1 conditional $\mathrm{KO}$ showed impaired $\mathrm{K}^{+}$clearance and axonal degeneration in the white matter (Larson et al., 2018; Schirmer et al., 2018). In Aldh1L1-CreERT2;Rosa26-tdTom mice, Cre 
recombination takes place almost exclusively in astrocytes (Figures 7A-7C) and only rarely in other cell types, such as oligodendrocytes or neurons (Winchenbach et al., 2016). The increase in Kir4.1 levels in N4-2 AstKO is similar in magnitude to that seen in N4-2 bKO (compare Figure 3Q and Figure 7F). These results indicate that N4-2 controls Kir4.1 levels mostly in astrocytes.

Like fluoxetine, GAP26 reverts the reduced power of $\gamma$-oscillations in N4-2 AstKOs (Figure 8). In principle, this effect of GAP26 could be due to blockade of either hemichannels or gap junctions - so far, it is not possible to block only gap junctions without affecting hemichannels by using blocking peptides or genetic approaches (e.g. knock-down or KO of Cx43). In our FRAP experiments, the calcein signal decayed without bleaching, likely because of calcein release through hemichannels. However, the time course of this decay ( $\sim 30 \mathrm{~min})$ was longer than that of FRAP ( $\sim 5 \mathrm{~min})$, indicating that gap junctions play a more prominent role than hemichannels in the astrocytic efflux of small solutes such as calcein, ions, or metabolic substrates.

In the N4-2 AstKO, the increased Cx43 levels could equally affect inter-astrocytic gap junctions composed of $\mathrm{Cx} 43$, gap junctions composed of $\mathrm{Cx} 43$ and $\mathrm{Cx} 36$ between astrocytes and neurons, and gap junctions composed of $\mathrm{Cx} 43$ and $\mathrm{Cx} 32$ between astrocytes and oligodendrocytes. Through upregulated gap junctions in N4-2 AstKO, the excitability of neurons might be influenced by the cumulative effect of enhanced $\mathrm{K}^{+}$buffering (Wallraff et al., 2006), increased lactate delivery to neurons (Clasadonte et al., 2017), and increased extracellular space volume (Pannasch et al., 2011), all of which might reduce the power of $\gamma$ oscillations. Of these possible explanations, we regard $\mathrm{K}^{+}$buffering as the most crucial. Even transient elevation of extracellular $\mathrm{K}^{+}$causes oscillatory activity in the $\gamma$-band frequency without much effect on other brain oscillation frequencies (LeBeau et al., 2002), which would be compatible with our results (Figures 8 and 9). 
$\gamma$-Oscillations precede epileptiform spike events (Ren et al., 2015), and visual stimuli that cause maximum power of $\gamma$-oscillations could trigger photosensitive epilepsy (Hermes et al., 2017; Perry et al., 2014). In this context, we found that missense mutations in N4-2 that were identified in patients with photosensitive epilepsy (S233L and H515P) cause increased E3 activity of N4-2 (Figure 2). This result indicates that the corresponding mutant N4-2 variants might cause reduced astrocytic expression of Kir4.1 and $\mathrm{Cx} 43$, reduced $\mathrm{K}^{+}$buffering, and correspondingly increased neuronal network activity, which could underlie the pathology of the patients carrying these N4-2 mutations (Bockenhauer et al., 2009; Djukic et al., 2007; Scholl et al., 2009; Wallraff et al., 2006) (Figure 10).

In conclusion, our study defines astrocytic ion channel proteostasis as an important determinant of astrocyte function and as a novel regulatory principle in neuronal network activity that may have major implications for the etiology of certain forms of epilepsy. 


\section{MATERIALS AND METHODS}

\section{Details of Antibodies}

\begin{tabular}{|c|c|c|}
\hline Antibodies & SOURCE & IDENTIFIER \\
\hline Guinea pig polyclonal ant-EAAT2 & Synaptic Systems & $\begin{array}{l}\text { Cat\#250204; } \\
\text { RRID:AB_2619958 }\end{array}$ \\
\hline Mouse monoclonal anti-beta-Tubulin & Sigma-Aldrich & $\begin{array}{l}\text { Cat \#T4026; } \\
\text { RRID:AB_477577 }\end{array}$ \\
\hline Rabbit polyclonal anti-GluA1 & Synaptic Systems & $\begin{array}{l}\text { Cat\#182003; } \\
\text { RRID:AB_2113441 }\end{array}$ \\
\hline Mouse monoclonal anti-Nedd4-1 & Becton Dickinson & $\begin{array}{l}\text { Cat\# 611481; } \\
\text { RRID:AB_398941 }\end{array}$ \\
\hline Rabbit polyclonal anti-Nedd4-2 & $\begin{array}{l}\text { Cell Signaling } \\
\text { Technologies }\end{array}$ & $\begin{array}{l}\text { Cat\#4013; } \\
\text { RRID:AB_1904063 }\end{array}$ \\
\hline Mouse monoclonal anti-GFP & Roche & $\begin{array}{l}\text { Cat \#11814460001; } \\
\text { RRID:AB_390913 }\end{array}$ \\
\hline Rabbit polyclonal anti-GFP & Synaptic Systems & $\begin{array}{l}\text { Cat\#132 003; } \\
\text { RRID:AB_1834147 }\end{array}$ \\
\hline Mouse monoclonal anti-PSD-95 & Becton Dickinson & $\begin{array}{l}\text { Cat\#610495; } \\
\text { RRID:AB_397861 }\end{array}$ \\
\hline
\end{tabular}




\begin{tabular}{|c|c|c|}
\hline Mouse monoclonal anti-RabGDI & Synaptic Systems & $\begin{array}{l}\text { Cat\#130 011; } \\
\text { RRID:AB_1966443 }\end{array}$ \\
\hline Rabbit polyclonal anti-Kir4.1 & Alomone & $\begin{array}{l}\text { Cat\#APC-035; } \\
\text { RRID:AB_2040120 }\end{array}$ \\
\hline Rabbit polyclonal anti-Connexin43 & Majoul et al., 2009 & \\
\hline Rabbit polyclonal anti-HA & Sigma-Aldrich & $\begin{array}{l}\text { Cat\#H6908; } \\
\text { RRID:AB_260070 }\end{array}$ \\
\hline Mouse monoclonal anti-ubiquitin (P4D1) & Santa Cruz & $\begin{array}{l}\text { Cat\#sc-8017; } \\
\text { RRID:AB_628423 }\end{array}$ \\
\hline Rabbit polyclonal anti-ubiquitin & Dako & $\begin{array}{l}\text { Cat\#Z0458; } \\
\text { RRID:AB_2315524 }\end{array}$ \\
\hline Mouse anti-NeuN & Millipore & Cat\#MAB377 \\
\hline Rabbit anti-Parvalbumin & Swant & Cat\#PV27 \\
\hline $\begin{array}{l}\text { Rabbit monoclonal anti-K63-linked } \\
\text { polyubiquitin (Apu3) }\end{array}$ & Merck Millipore & $\begin{array}{l}\text { Cat } \# 05-1308 ; \\
\text { RRID:AB_1587580 }\end{array}$ \\
\hline
\end{tabular}




\begin{tabular}{|c|c|c|}
\hline $\begin{array}{l}\text { Rabbit monoclonal anti-K48-linked } \\
\text { polyubiquitin (Apu2) }\end{array}$ & Merck Millipore & $\begin{array}{l}\text { Cat } \# 05-1307 ; \\
\text { RRID:AB_1587578 }\end{array}$ \\
\hline Rabbit polyclonal anti-S100 antibody & $\begin{array}{l}\text { Agilent } \\
\text { Technologies }\end{array}$ & $\begin{array}{l}\text { Cat\#Z0311; } \\
\text { RRID:AB_1001338 } \\
3\end{array}$ \\
\hline Goat anti-Mouse IgG-HRP & Dianova & $\begin{array}{l}\text { Cat\#115-035-146; } \\
\text { RRID:AB_2307392 }\end{array}$ \\
\hline Goat anti-Rabbit IgG-HRP & Dianova & $\begin{array}{l}\text { Cat\#111-035-144; } \\
\text { RRID:AB_2307391 }\end{array}$ \\
\hline Goat anti-Mouse IgG-IRDye 800 & $\begin{array}{l}\text { Rockland } \\
\text { Immunochemicals }\end{array}$ & $\begin{array}{l}\text { Cat\#610-132-121; } \\
\text { RRID:AB_220125 }\end{array}$ \\
\hline Goat anti-Rabbit IgG-IRDye 800 & $\begin{array}{l}\text { Rockland } \\
\text { Immunochemicals }\end{array}$ & $\begin{array}{l}\text { Cat\#611-132-122; } \\
\text { RRID:AB_220152 }\end{array}$ \\
\hline Goat anti-Mouse IgG-IRDye700 & $\begin{array}{l}\text { Rockland } \\
\text { Immunochemicals }\end{array}$ & $\begin{array}{l}\text { Cat\#610-130-121; } \\
\text { RRID:AB_220121 }\end{array}$ \\
\hline Goat anti-Rabbit IgG-IRDye700 & $\begin{array}{l}\text { Rockland } \\
\text { Immunochemicals }\end{array}$ & $\begin{array}{l}\text { Cat\#611-130-122; } \\
\text { RRID:AB_220148 }\end{array}$ \\
\hline
\end{tabular}




\begin{tabular}{|l|l|l|}
\hline Goat anti-Guinea pig IgG-IRDye700 & Rockland & Cat\#606-130-129; \\
& Immunochemicals & RRID:AB_220105 \\
\hline Goat anti-Mouse IgG-Alexa555 & Thermo Fisher & Cat\#A-21424; \\
\hline Goat anti-Rabbit IgG-Alexa488 & Thermo Fisher & Cat\#A-11008; \\
& & RRID:AB_143165 141780 \\
\hline
\end{tabular}

\section{Animal Experiments}

All experiments using mice were performed in Max Planck Institute of Experimental Medicine in compliance with the guidelines for the welfare of experimental animals approved by the State Government of Lower Saxony (Niedersächsisches Landesamt für Verbraucherschutz und Lebensmittelsicherheit Permission 33.9-42502-04-13/1359, 33.1942502-04-15/1954, and 33.19-42502-04-16/2173) and the Max Planck Society (comparable to National Institute of Health Guidelines). For all animal experiments, mice backcrossed with the C57BL/6N more than ten times were used. Nedd4-2 ${ }^{\mathrm{f} / \mathrm{f}}$;Aldh1l1-CreERT2 and Nedd4$2^{\mathrm{f} / \mathrm{f}}$ male mice were injected with $50 \mu \mathrm{l}$ of $10 \mathrm{mg} / \mathrm{ml}$ tamoxifen for five consecutive days starting at P13 to P15. At the age of five weeks, mice were anesthetized by intraperitoneal injection of urethane (1.3-1.5 $\mathrm{mg} / \mathrm{kg}$ body weight) prior to the in vivo recording.

\section{In vitro Recording of $\gamma$-Oscillations}

Recordings of LFPs from acute hippocampal slices and the induction of oscillations were performed as previously described (Ripamonti et al., 2017). The frequency at maximum power peak and average power of oscillations were determined between $25-45 \mathrm{~Hz}$. For rescue 
experiments performed under conditions of Kir4.1 inhibition, the baseline spectrum was recorded in artificial cerebrospinal fluid (ACSF) and $\gamma$-oscillation was induced with Kainate Solution (100 nM Kainate in ACSF) in the presence of $30 \mu \mathrm{M}$ Fluoxetine, which inhibits Kir4.1 channel (Sigma, Cat\#PHR1394-1G). Since GAP26 has a larger molecular weight than Fluoxetine, it is generally required to incubate the tissue with this inhibitor prior to functional experiments to achieve efficient penetration of GAP26 into the tissue. For rescue experiments performed under conditions of $\mathrm{Cx} 43$ inhibition, slices were pre-incubated for 45-60 $\mathrm{min}$ in ACSF containing $50 \mu \mathrm{M}$ the $\mathrm{Cx} 43$ inhibitor GAP26, the baseline spectrum was measured in the same solution, and $\gamma$-oscillation was induced by Kainate Solution containing $50 \mu \mathrm{M}$ GAP26. Each set of pharmacological rescue experiments (i.e. recordings from N4-2 CTL, N4-2 AstKO, and N4-2 AstKO treated with a blocker) was performed as a single sets of recording experiments.

\section{Quantitative Mass Spectrometry}

SM fractions were purified as previously reported (Mizoguchi et al., 1989). Proteins in pooled SM fractions from N4-1/2 CTL, N4-1/2 bDKO, and a 1:1 mixture of both were separated on precast gradient gels and visualized by colloidal Coomassie staining. Proteins in the gel were subjected to in-gel digestion, and isobaric peptide labeling as described (Schmidt et al., 2013). The iTRAQ Reagent 4plex Kit (Sciex, Cat\#4352135) was used to label the tryptic peptides derived from the different SM fractions as follows: iTRAQ114, mixture of equal volumes of all three samples to be compared; iTRAQ115, 1:1 mixture of N4-1/2 CTL and N4-1/2 bDKO; iTRAQ116, N4-1/2 CTL; iTRAQ117, N4-1/2 bDKO (forward labeling). To control for reagent-specific labeling artifacts, a replicate experiment was performed, in which iTRAQ channels 116 and 117 were switched (reverse labeling). The iTRAQ114/115 channels served as internal controls for normalization and quality control of the iTRAQ reporter ion signals. Labeled peptides were pooled and analyzed by liquid chromatography 
coupled to electrospray mass spectrometry using a LTQ Orbitrap Velos hybrid mass spectrometer (Thermo Fisher Scientific) operated in data-dependent mode with higher-energy C-trap fragmentation as described (Schmidt et al., 2013). MS raw data were processed with the MaxQuant software (version 1.3.0.5) and peak lists were searched with the built-in Andromeda search engine (Cox and Mann, 2008) against UniProtKB M. Musculus protein database (downloaded 2013-05-14) supplemented with common contaminants and concatenated with the reverse sequences of all entries. Search parameters were set to carbamidomethylation of cysteines as fixed, and oxidation of methionine and N-terminal acetylation as variable modifications. Trypsin without proline restriction was specified as digestion enzyme and up to two missed cleavages were allowed. The precursor and the fragment ion mass tolerance was set to $7 \mathrm{ppm}$ and $20 \mathrm{ppm}$, respectively. A minimal length of six amino acids per identified peptide was required for protein identification. The false discovery rate was set to $1 \%$ at both peptide and protein level. The command 're-quantify' was enabled, and 'keep low scoring versions of identified peptides' was disabled. Statistical analysis was performed with the Perseus bioinformatics platform to calculate 'Significance B' (Tyanova et al., 2016). For candidate selection, only proteins with minimum two identified peptides in total (at least one unique) were considered and consistent up- or down-regulation in both experiments (forward and reverse labeling) was required. Up-regulated proteins with a 'Significance B' value below 0.05 in both experiments were considered as potential substrates.

\section{Quantitative Western Blotting}

Quantitative Western blotting was performed as previously reported (Hsia et al., 2014). Signals were normalized to Tubulin levels. Brain lysates from $G l u A 1^{-/-}$and the control mice were provided by Drs. Thorsten Bus and Rolf Sprengel (Max Planck Institute for Medical Research, Heidelberg, Germany) (Zamanillo et al., 1999). All values from one set of 
experiments were normalized to the average value of CTL before statistical analyses. Samples were excluded from statistical analyses when bands in Western blotting were not isolated or were obviously deformed. Averages, SEMs, p-values, and statistical tests are documented in Tables S2, S3, and S4. Sample numbers are documented within legends of each figure.

\section{Quantitative RT-PCR}

Cortices were dissected from CTL and N4-1/2 bDKO mice with tools cleaned with $70 \%$ ethanol (Sigma) and RNA-Zap (Thermo Fisher), and flash-frozen in liquid nitrogen. Samples were homogenized in $600-1000 \mu \mathrm{l}$ of Trizol (Thermo Fisher) in $2 \mathrm{ml}$ eppendorf tubes by using Ultra Turrax homogenizer (IKA Labtechnik) for 40 seconds and incubated at the room temperature for $3 \mathrm{~min}$. Samples were centrifuged at 10,000 rpm for $2 \mathrm{~min}$, and supernatants were collected mixed with the equal volume of ethanol. Mixtures were applied onto Zymo Spin Columns to purify RNA (Direct-zol, RNA MiniPrep Plus, Zymo, Cat\#R2001). RNA estimation, RT-PCR, and qPCR were performed based on a published protocol (Nolan et al., 2006). Kir4.1 and Cx43 levels normalized to HPRT1 level were calculated based on $2^{-}$ ${ }^{\Delta \Delta C(T)}$ method (Livak and Schmittgen, 2001). All values from one set of experiments were normalized to the average value of CTL before statistical analyses. Averages, SEMs, pvalues, and statistical tests are documented in Table S2. Animal numbers are documented in the end of the legend for Figures 3.

Kir4.1 Forward: 5'-AGTCTTGGCCCTGCCTGT -3', Reverse: 5'-AGCGACCGACGTCATCTT -3';

Cx43 Forward: 5'-TCCTTTGACTTCAGCCTCCA -3',

Reverse: 5'-CCATGTCTGGGCACCTCT -3'; 
HPRT1 Forward: 5'-GCTTGCTGGTGAAAAGGACCTCTCGAAG -3', Reverse: 5'-CCCTGAAGTACTCATTATAGTCAAGGGCAT -3'

Pre-incubation

Step 1: $95^{\circ} \mathrm{C}$ for $10 \mathrm{~min}$

Amplification

Step 2: $95^{\circ} \mathrm{C}$ for 10 seconds

Step 3: $60^{\circ} \mathrm{C}$ for 30 seconds

Step 4: $72^{\circ} \mathrm{C}$ for $1 \mathrm{~min}$ (45 cycles from Step 2 to 3 )

Cooling

Step 5: $40^{\circ} \mathrm{C}$ for 10 seconds

\section{In vitro Ubiquitination Assay}

For in vitro ubiquitination assays, recombinant mouse E1 (Addgene plasmid \# 32534), E2 (pGEX4T-1-UbcH5b), and E3s (pDEST-Nedd4-2) were expressed in BL21 Rosetta 2 (DE3) E.coli and purified according to published protocols (Albert et al., 2002; Carvalho et al., 2012; Persaud et al., 2009) with slight modifications. Reaction mixture containing $2 \mathrm{mM}$ ATP, $1 \mu$ M His-Ubiquitin (Boston Biochem, Cat\#U-530), 50 nM mouse E1 enzyme, $150 \mathrm{nM}$ E2 enzyme (UbcH5b), 300 nM E3 enzyme (Nedd4-2 WT, S233L,or H515P) in Reaction Buffer [50 mM Tris-Cl pH 7.5 at $37^{\circ} \mathrm{C}, 100 \mathrm{mM} \mathrm{NaCl}, 5 \mathrm{mM} \mathrm{MgCl}_{2}, 1 \mathrm{mM}$ DTT, $0.05 \%$ (w/v) Tween 20] was incubated at $37^{\circ} \mathrm{C}$. Reactions were stopped by addition of $1 \mathrm{X}$ Laemmli Buffer, and polyubiquitin chain formation was detected by Western blotting using the anti- 
ubiquitin antibody. Signals from secondary antibodies were acquired and quantified by an Odyssey Imaging System (LI-COR). For the study of the time course of in vitro ubiquitination reaction in Figure $2 \mathrm{~B}$, the signal at the molecular weight of $\sim 9 \mathrm{kDa}$ (i.e. the signal from free ubiquitin) at each time point was expressed as the level relative to free ubiquitin at the time point of $0 \mathrm{sec}$ (Figure 2B, left panel). Signals from polyubiquitin chains detected by the anti-ubiquitin antibody were quantified from the top of the SDS-PAGE separating gel to the approximate molecular weight of $50 \mathrm{kDa}$. The same size of the region of interest was applied for the quantification for one set of experiments. Given that the level of free ubiquitin is close to $0 \%$ at $300 \mathrm{sec}$ in the left panel in Figure 2B, signals from polyubiquitin chains at each time point were expressed as the level relative to the time point of $300 \mathrm{sec}$ in the right panel of Figure 2B. For statistical analyses of free ubiquitin in top panels of Figures 2D and 2F, signals from free ubiquitin was normalized to Nedd4-2 level. Reduction of normalized signals at $150 \mathrm{sec}$ relative to the level at $0 \mathrm{sec}$ was expressed as depletion of free ubiquitin. For statistics of polyubiquitin chains in Figures 2D and 2F, signals from polyubiquitin chains were normalized to the level of Nedd4-2 first, and then further normalized to the average value of the control (N4-2 WT). Averages, SEMs, p-values, and statistical tests are documented in Table S2. Sample numbers are documented in the end of the legend for Figure 2. Experiments were done at least twice.

\section{Cell Culture}

HEK293FT cells were obtained from Thermo Fisher (Cat\#R70007) and maintained in Dulbecco's Modified Eagle Medium (DMEM, Thermo Fisher, Cat\#41966-029) supplemented by $10 \%$ Fetal Bovine Serum (FBS, Thermo Fisher, Cat\#10500-064), $100 \mathrm{U} / \mathrm{ml}$ penicillin and streptomycin (Thermo Fisher, Cat\#15140-122), and $2 \mathrm{mM}$ glutamine (Thermo Fisher, Cat\#25030-024) in $37^{\circ} \mathrm{C}$ incubator with $5 \% \mathrm{CO}_{2}$. For primary astrocyte culture, mutant mice 
backcrossed with the C57BL/6N more than ten times were used. Cortical astrocyte culture was prepared according to previously published papers (Burgalossi et al., 2012).

\section{Lentivirus Infection and Dynasore Treatment}

Lentiviral vectors were produced in HEK293FT cells according to our previous publication (Hsia et al., 2014). Viruses were infected 16-24 hours after plating astrocytes, which were harvested 5 days after infection. Viral titers were adjusted to express either recombinant N4-2 WT or the N4-2 C/S at the level comparable to the endogenous protein level (compare first, third, and fourth lanes in second Western blotting panels in Figure 4G). Under these conditions, $\sim 90 \%$ astrocytes were transduced. $100 \mu \mathrm{M}$ dynasore was applied to astrocytes 40 48 hours after plating astrocytes. Cells were harvested 12 hours after the application of dynasore. Astrocytes were harvested in Laemmli buffer containing $5 \mathrm{mM} \mathrm{MgCl}_{2}$ and 16.7 units/ $\mu$ l of Benzonase.

\section{In vivo Ubiquitination Assay}

In order to show ubiquitination of substrates in vivo, cell-based ubiquitination assay was performed using HEK293FT cells as previously reported (Kawabe et al., 2010). For the ubiquitination assay using primary cultured astrocytes, primary cultured cortical astrocytes were prepared from N4-1/2 bDKO and N4-1/2 CTL mice at the age of postnatal day 0 (P0) or 2. Astrocytes were infected with HA-tagged Kir4.1- or Cx43-expression lentivirus. 5 days after infection, cultured astrocytes were washed with PBS containing $10 \mathrm{mM}$ Nethylmaleimide at least 5 times. Subsequent procedures were performed with the same protocol as the in vivo ubiquitination assay using HEK293FT cells. Experiments were performed at least twice.

\section{In vitro Binding Assay}


pGex6P-1 plasmids to express truncated mutants of mouse Nedd4-2 (GenBank Accession Nr. NM_001114386) encode amino acid sequences of Nedd4-2 as listed below;

1) pGex6P-1 Nedd4-2 C2+WW1 (1-326 aa)

2) pGex6P-1 Nedd4-2 WW1 (141-326 aa)

3) pGex6P-1 Nedd4-2 WW1+WW2 (141-475 aa)

4) pGex6P-1 Nedd4-2 WW2 (321-475 aa)

5) pGex6P-1 Nedd4-2 WW3+WW4 (474-595 aa)

6) pGex6P-1 Nedd4-2 WW2+WW3+WW4 (321-595 aa)

Proteins were expressed and purified from BL21 Rosetta 2 (DE3) E. coli cells and bindings of Kir4.1-HA and Cx43-HA were studied as previously reported (Kawabe et al., 2010).

\section{Patch Clamp Recordings in Astrocytes}

Brain slices with $300 \mu \mathrm{m}$ thickness were prepared as for extracellular recordings. Astrocytes were labeled with sulforhodamine $101\left(1 \mu \mathrm{M}\right.$ in $\left.\mathrm{ACSF}, 34^{\circ} \mathrm{C}, 20 \mathrm{~min}\right)$ followed by washing with $\operatorname{ACSF}\left(10 \mathrm{~min}, 34^{\circ} \mathrm{C}\right)$. Later, brain slices were kept at room temperature until recordings. For whole-cell recordings, slices were transferred to a custom-made recording chamber on an upright microscope (BX51W1, Olympus). Sulforhodamine-labelled astrocytes were identified using epiflurorescence illumination (U-RFL-T, Olympus). Once cells were identified, astrocytes were patched with glass pipettes (4-6 M $\Omega$ ) filled with intracellular solution (100 mM KCl, 50 mM K-gluconate, 10 mM HEPES, 0.1 mM EGTA, 0.3 mM GTP, $4 \mathrm{mM}$ Mg-ATP, $0.4 \%$ Biocytin, $\mathrm{pH}$ 7.4) under transmitted light (TH4-200, Olympus, AxioCam MRm, Zeiss). Whole-cell voltage-clamp recordings were obtained using EPC10 amplifier (HEKA System). Membrane currents were low-pass filtered at $2 \mathrm{kHz}$ and digitized 
at $20 \mathrm{kHz}$. Astrocytes were voltage-clamped to $-80 \mathrm{mV}$ and voltage-current plots were obtained using $10 \mathrm{mV}$ voltage steps ranging from -120 to $+40 \mathrm{mV}$ or from -100 to $+40 \mathrm{mV}$. For the rescue experiments, $30 \mu \mathrm{M}$ fluoxetine or $100 \mu \mathrm{M}$ barium chloride was included in ACSF during the recording.

\section{FRAP Experiments}

Astrocytes were plated on a glass bottom culture dish (ibidi, Cat\#80427) in astrocyte culture medium. The humidity, temperature, and $\mathrm{CO}_{2}$ concentration of the cultures were maintained at $95-98 \%, 37^{\circ} \mathrm{C}$, and $5 \%$ respectively during FRAP imaging experiments. For the purpose of labeling, astrocytes were incubated with calcein-AM (Sigma, Cat\#17783-1MG) for $30 \mathrm{~min}$ to one hour prior to imaging to allow cells to uptake the fluorophore. After removing the calcein-AM-containing medium and washing cells with fresh medium three times, calcein in the astrocytes was bleached and images were obtained at $100 \mathrm{msec}$ intervals in the first 15 sec after bleaching and subsequently at $5 \mathrm{sec}$ intervals with a Nikon Eclipse Ti microscope equipped with hardware autofocus (Perfect Focus), a spinning disk confocal unit (W1 Yokogawa), and an Andor FRAPPA unit used for bleaching. Fluorescence from the cultured astrocytes was observed with 40x oil immersion objective lens (1.30 NA). At each time point $(\mathrm{t})$, background signal intensities $\left[\mathrm{I}_{\mathrm{back}}(\mathrm{t})\right]$ and photobleached or non-bleached reference cell intensities $\left[\mathrm{I}_{\text {frap }}(\mathrm{t})\right.$ or $\left.\mathrm{I}_{\text {ref }}(\mathrm{t})\right]$ were monitored during imaging and then quantified. Bleached time point is defined as tb. Sampling number at bleach time point is defined as $\mathrm{n}_{\text {bleach. }}$ FRAP was estimated as follows;

1) Normalized signal intensity from beached cells $(\operatorname{Infrap}(t))$

$\operatorname{Infrap}(\mathrm{t})=\left(\mathrm{I}_{\text {frap }}(\mathrm{t})-\mathrm{I}_{\text {back }}(\mathrm{t})\right) /\left(\mathrm{I}_{\text {ref }}(\mathrm{t})-\mathrm{I}_{\text {back }}(\mathrm{t})\right)$

2) Full range of bleaching $\left(\mathrm{I}_{\text {fullbleach }}\right)$ 


$$
\mathrm{I}_{\text {fullbleach }}=\sum_{\mathrm{t}=0}^{\mathrm{t}=\mathrm{tb}-1} \operatorname{Infrap}(\mathrm{t}) / \text { nbleach }-\operatorname{Infrap}(\mathrm{tb})
$$

3) $\quad$ FRAP efficiency $(\%)$ at $\mathrm{t}=(\operatorname{Infrap}(\mathrm{t})-\operatorname{Infrap}(\mathrm{tb})) / \mathrm{I}_{\text {fullbleach }} \mathrm{x} 100$

Averages, SEMs, p-values, and statistical tests are documented in Table S2. Sample numbers are documented within the end of the legend for Figure 6. Experiments were performed at least twice by an observer blinded to genotypes.

\section{In vivo Recording of $\gamma$-Oscillations}

Five-week-old mice were anesthetized with urethane $(1.3-1.5 \mathrm{mg} / \mathrm{kg}$ ) and placed on a stereotaxic frame at $36{ }^{\circ} \mathrm{C}$ (ATC1000, WPI, Cat\#61805) while monitoring. A craniotomy of $1 \times 1 \mathrm{~mm}$ was made centered at $2 \mathrm{~mm}$ posterior from lambda and $2.6 \mathrm{~mm}$ lateral from the midline. A linear multielectrode array (Neuronexus, Cat\#A1-16-3mm-50-177) coated with DiI was inserted from the cortical surface orthogonally and placed at $2.5 \mathrm{~mm}$ from brain surface to target CA3. Signals from field potentials were pre-amplified (Neuralynx, Cat\#HS18) and sent to an acquisition board (Digital Lynx 4SX, Neuralynx, Cat\#DL-4SX) at a sampling rate of $32 \mathrm{kHz}$. After $30 \mathrm{~min}$ of recording of the baseline activity, kainate (20 $\mathrm{mg} / \mathrm{kg}$, BioTrend, Cat\#BN0281) was injected intraperitoneally and the recordings were extended for 60 minutes. After recordings, the brain was fixed in 4\% paraformaldehyde. The position of the electrode was validated based on signals from DiI in the brain tissue after sectioning.

Local field potentials were analyzed in MATLAB (Mathworks). The signal was filtered (0.7$400 \mathrm{~Hz}$ ) and downsampled to $1 \mathrm{kHz}$. Power was calculated with the multitaper method (Chronux Package, http://chronux.org, 5 tapers, time bandwidth product of 3 seconds and 0.2 seconds overlap). The data were normalized by dividing the power spectra of each individual 
time point after kainate injection by the average power spectra of the baseline activity. The range of the $\gamma$-band was $25-45 \mathrm{~Hz}$. Values between groups across time were compared using a repeated measures two-way ANOVA (genotype $\mathrm{x}$ time), followed by post-hoc Fisher's Least Significant Difference corrections. Averages, SEMs, p-values, and statistical tests are documented in Table S5. Sample numbers are documented in figure legend for Figure 9.

\section{Statistical Analysis}

For statistical analyses, Graph Pad Prism 5 or MATLAB software was used. All results were expressed as mean \pm SEM. Two independent groups were analyzed by Student's t-test, where significance was defined as $p<0.05$. For electrophysiological experiments, data were acquired by observers blinded to genotype except for the recue experiment in Figures 5, 7H-7M, and 8, where we applied fluoxetine or GAP26 only to N4-2 bKO or AstKO samples. One-way ANOVA test was applied for the comparison of one parameter between three or more groups. When relatively small difference was expected (i.e. partial rescues of reduced $\gamma$-oscillation in Nedd4-2 AstKO by GAP26 or Fluoxetin), Newman-Keuls post-hoc test applied for comparison of two groups. Otherwise, Tukey post-hoc test was used. For comparisons of more than two parameters between two groups in Figure 9, two-way ANOVA with LSD post-hoc test was applied.

\section{Quantification of Electrophysiological Recording using Acute Slices}

For field recording experiments, absolute powers and frequencies of $\gamma$-oscillations were analyzed statistically. For patch clamp experiments, absolute membrane currents and resistances were analyzed. All sets of experiments were performed twice with two or more pairs of animals. Outliers were excluded from statistical analyses using Graph Pad Prism software. Datasets with a serial decline in the baseline of the local field potential were also excluded from analyses. Averages, SEMs, p-values, and statistical tests are documented in 
Table S2, S3, and S4. Numbers of recorded cells and acute slices are documented within legends of Figure 1, 5, 7, and 8.

\section{Quantitative Immunohistochemistry}

Five-week old mice were perfused intracardially with $4 \%$ paraformaldehyde and the brains were dissected for overnight post-fixation with $4 \%$ paraformaldehyde at $4{ }^{\circ} \mathrm{C}$. After washing the brains with PBS several times, they were cryoprotected with $30 \%$ sucrose. $15 \mu \mathrm{m}$ hippocampal sections were prepared using a cryostat (Leica CM3050 S) and were stained with primary and secondary antibodies. Signals from secondary antibodies were acquired by Leica TCS-SP2 confocal microscopy using $20 \mathrm{X}$ oil immersion objective lens with the digital zoom of 1X. Averages, SEMs, p-values, and statistical tests are documented in Table S2. Experiments were performed with two pairs of animals. Numbers of sections are documented in the legends of Figure 7.

\section{Summary of Supplemental Materials}

Table S1: Details of proteome data. Related to Figure 3E. Highlighted in magenta and green are proteins up- or downregulated in N4-1/2 bDKO for both forward and reverse experiments significantly. In the tab 'all proteins simplified', informations of all identified proteins are provided. In the tab 'all proteins raw data', original data of all identified proteins are listed.

Table S2: Details of Student's t-tests. Related to Figures 1C, 1D, 1G, 1H, 2D, 2F, 3C, 3G, 3H, 3J, 3K, 3L, 3N, 3O, 3Q, 3R, 6E, 7E, 7F, 7G, 7O, and 7P.

Table S3: Details of multiple comparison tests. Related to Figures 4G, 4H, 5E, 5F, 7L, 7M, $8 \mathrm{D}, 8 \mathrm{E}, 8 \mathrm{I}$, and $8 \mathrm{~J}$.

Table S4: Details of post-hoc tests in ANOVA tests. Related to Figures 4G, 4H, 5E, 5F, 7L, $7 \mathrm{M}, 8 \mathrm{D}, 8 \mathrm{E}, 8 \mathrm{I}$, and $8 \mathrm{~J}$. 
bioRxiv preprint doi: https://doi.org/10.1101/683441; this version posted July 4, 2019. The copyright holder for this preprint (which was not certified by peer review) is the author/funder. All rights reserved. No reuse allowed without permission.

Table S5: Details of repeated measures two-way ANOVA test. Related to Figure 9C. 


\section{ACKNOWLEDGEMENTS}

This work was supported by the German Research Foundation (SPP1365/KA3423/1-1 and KA3423/3-1, HK), the Fritz Thyssen Foundation (HK), and the JSPS KAKENHI Grant Number 15K21769 (HK), and The Mother and Child Health Foundation (Grant Number 29-9, HK). We thank Nils Brose for his support, advice, and comments on the manuscript, Vincent O'Conner, Erinn Gideons, James Daniel, and Alexandros Poulopoulos for their helpful comments and critical reviews of the manuscript, and Klaus Nave for his support. We are grateful to Fritz Benseler, Klaus-Peter Hellmann, Bernd Hesse-Niessen, Ivonne Thanhäuser, Dayana Warnecke, Christiane Harenberg, Maik Schlieper, Lars van Werven, and Dörte Hesse for excellent technical support, and to the Animal Facilities of the Max Planck Institute of Experimental Medicine for the maintenance of mouse colonies. We thank Johannes Hirrlinger, Frank Kirchhoff, Nicola Strenzke, and Gulnara Yamanbaeva for their advice. We thank Kevin R. Jones and Hongkui Zeng for providing EMX-Cre and ROSA26-tdTom mouse lines respectively, Thorsten Bus and Rolf Sprengel for providing brain tissue and tissue extracts of control and Grial $1^{-/}$mice, Jorge E. Azevedo, Daniela Rotin, David Baltimore, Richard Huganir, Didier Trono, Robin Shaw, and Marc Timmers for providing plasmids. The authors declare no competing financial interests. 


\section{AUTHOR CONTRIBUTIONS}

BA: conceptualization, project administration, investigation, validation, formal analysis, writing-original draft, and writing-review and editing; AJ, HCS, SK, JW, ÖK, MS, GLW, VKC, IM, MAH, RG, SMW: investigation, validation, and formal analysis; AP, MM, LdH, HU, OJ, GS, JSR: conceptualization; HK: supervision, conceptualization, provided funding for the research, project administration, writing-review and editing. 


\section{REFERENCES}

Abbaci, M., M. Barberi-Heyob, J.R. Stines, W. Blondel, D. Dumas, F. Guillemin, and J. Didelon. 2007. Gap junctional intercellular communication capacity by gap-FRAP technique: a comparative study. Biotechnol J. 2:50-61.

Acconcia, F., S. Sigismund, and S. Polo. 2009. Ubiquitin in trafficking: the network at work. Exp Cell Res. 315:1610-1618.

Albert, T.K., H. Hanzawa, Y.I. Legtenberg, M.J. de Ruwe, F.A. van den Heuvel, M.A. Collart, R. Boelens, and H.T. Timmers. 2002. Identification of a ubiquitin-protein ligase subunit within the CCR4-NOT transcription repressor complex. EMBO J. 21:355-364.

Allen, A.S., S.F. Berkovic, P. Cossette, N. Delanty, D. Dlugos, E.E. Eichler, M.P. Epstein, T. Glauser, D.B. Goldstein, Y. Han, E.L. Heinzen, Y. Hitomi, K.B. Howell, M.R. Johnson, R. Kuzniecky, D.H. Lowenstein, Y.F. Lu, M.R. Madou, A.G. Marson, H.C. Mefford, S. Esmaeeli Nieh, T.J. O'Brien, R. Ottman, S. Petrovski, A. Poduri, E.K. Ruzzo, I.E. Scheffer, E.H. Sherr, C.J. Yuskaitis, B. Abou-Khalil, B.K. Alldredge, J.F. Bautista, S.F. Berkovic, A. Boro, G.D. Cascino, D. Consalvo, P. Crumrine, O. Devinsky, D. Dlugos, M.P. Epstein, M. Fiol, N.B. Fountain, J. French, D. Friedman, E.B. Geller, T. Glauser, S. Glynn, S.R. Haut, J. Hayward, S.L. Helmers, S. Joshi, A. Kanner, H.E. Kirsch, R.C. Knowlton, E.H. Kossoff, R. Kuperman, R. Kuzniecky, D.H. Lowenstein, S.M. McGuire, P.V. Motika, E.J. Novotny, R. Ottman, J.M. Paolicchi, J.M. Parent, K. Park, A. Poduri, I.E. Scheffer, R.A. Shellhaas, E.H. Sherr, J.J. Shih, R. Singh, J. Sirven, M.C. Smith, J. Sullivan, L. Lin Thio, A. Venkat, E.P. Vining, G.K. Von Allmen, J.L. Weisenberg, P. Widdess-Walsh, and M.R. Winawer. 2013. De novo mutations in epileptic encephalopathies. Nature. 501:217-221.

Araque, A., R.P. Sanzgiri, V. Parpura, and P.G. Haydon. 1998. Calcium elevation in astrocytes causes an NMDA receptor-dependent increase in the frequency of miniature synaptic currents in cultured hippocampal neurons. J Neurosci. 18:68226829.

Bockenhauer, D., S. Feather, H.C. Stanescu, S. Bandulik, A.A. Zdebik, M. Reichold, J. Tobin, E. Lieberer, C. Sterner, G. Landoure, R. Arora, T. Sirimanna, D. Thompson, J.H. Cross, W. van't Hoff, O. Al Masri, K. Tullus, S. Yeung, Y. Anikster, E. Klootwijk, M. Hubank, M.J. Dillon, D. Heitzmann, M. Arcos-Burgos, M.A. Knepper, A. Dobbie, W.A. Gahl, R. Warth, E. Sheridan, and R. Kleta. 2009. Epilepsy, ataxia, sensorineural deafness, tubulopathy, and KCNJ10 mutations. $N$ Engl J Med. 360:1960-1970.

Burgalossi, A., S. Jung, K.N. Man, R. Nair, W.J. Jockusch, S.M. Wojcik, N. Brose, and J.S. Rhee. 2012. Analysis of neurotransmitter release mechanisms by photolysis of caged $\mathrm{Ca}(2)(+)$ in an autaptic neuron culture system. Nat Protoc. 7:1351-1365.

Cardin, J.A., M. Carlen, K. Meletis, U. Knoblich, F. Zhang, K. Deisseroth, L.H. Tsai, and C.I. Moore. 2009. Driving fast-spiking cells induces gamma rhythm and controls sensory responses. Nature. 459:663-667.

Carvalho, A.F., M.P. Pinto, C.P. Grou, R. Vitorino, P. Domingues, F. Yamao, C. SaMiranda, and J.E. Azevedo. 2012. High-yield expression in Escherichia coli and purification of mouse ubiquitin-activating enzyme E1. Mol Biotechnol. 51:254-261.

Chaytor, A.T., W.H. Evans, and T.M. Griffith. 1997. Peptides homologous to extracellular loop motifs of connexin 43 reversibly abolish rhythmic contractile activity in rabbit arteries. J Physiol. 503 ( Pt 1):99-110.

Chever, O., B. Djukic, K.D. McCarthy, and F. Amzica. 2010. Implication of Kir4.1 channel in excess potassium clearance: an in vivo study on anesthetized glial-conditional Kir4.1 knock-out mice. J Neurosci. 30:15769-15777. 
Clasadonte, J., J. Dong, D.J. Hines, and P.G. Haydon. 2013. Astrocyte control of synaptic NMDA receptors contributes to the progressive development of temporal lobe epilepsy. Proc Natl Acad Sci U S A. 110:17540-17545.

Clasadonte, J., E. Scemes, Z. Wang, D. Boison, and P.G. Haydon. 2017. Connexin 43Mediated Astroglial Metabolic Networks Contribute to the Regulation of the SleepWake Cycle. Neuron. 95:1365-1380 e1365.

Cox, J., and M. Mann. 2008. MaxQuant enables high peptide identification rates, individualized p.p.b.-range mass accuracies and proteome-wide protein quantification. Nat Biotechnol. 26:1367-1372.

Cui, Y., Y. Yang, Z. Ni, Y. Dong, G. Cai, A. Foncelle, S. Ma, K. Sang, S. Tang, Y. Li, Y. Shen, H. Berry, S. Wu, and H. Hu. 2018. Astroglial Kir4.1 in the lateral habenula drives neuronal bursts in depression. Nature. 554:323-327.

Dibbens, L.M., J. Ekberg, I. Taylor, B.L. Hodgson, S.J. Conroy, I.L. Lensink, S. Kumar, M.A. Zielinski, L.A. Harkin, G.R. Sutherland, D.J. Adams, S.F. Berkovic, I.E. Scheffer, J.C. Mulley, and P. Poronnik. 2007. NEDD4-2 as a potential candidate susceptibility gene for epileptic photosensitivity. Genes Brain Behav. 6:750-755.

Djukic, B., K.B. Casper, B.D. Philpot, L.S. Chin, and K.D. McCarthy. 2007. Conditional knock-out of Kir4.1 leads to glial membrane depolarization, inhibition of potassium and glutamate uptake, and enhanced short-term synaptic potentiation. $J$ Neurosci. 27:11354-11365.

Fang, N.N., G.T. Chan, M. Zhu, S.A. Comyn, A. Persaud, R.J. Deshaies, D. Rotin, J. Gsponer, and T. Mayor. 2014. Rsp5/Nedd4 is the main ubiquitin ligase that targets cytosolic misfolded proteins following heat stress. Nat Cell Biol. 16:1227-1237.

Farmer, W.T., T. Abrahamsson, S. Chierzi, C. Lui, C. Zaelzer, E.V. Jones, B.P. Bally, G.G. Chen, J.F. Theroux, J. Peng, C.W. Bourque, F. Charron, C. Ernst, P.J. Sjostrom, and K.K. Murai. 2016. Neurons diversify astrocytes in the adult brain through sonic hedgehog signaling. Science. 351:849-854.

Firsov, D., L. Schild, I. Gautschi, A.M. Merillat, E. Schneeberger, and B.C. Rossier. 1996. Cell surface expression of the epithelial $\mathrm{Na}$ channel and a mutant causing Liddle syndrome: a quantitative approach. Proc Natl Acad Sci U S A. 93:15370-15375.

Gloveli, T., T. Dugladze, S. Saha, H. Monyer, U. Heinemann, R.D. Traub, M.A. Whittington, and E.H. Buhl. 2005. Differential involvement of oriens/pyramidale interneurones in hippocampal network oscillations in vitro. J Physiol. 562:131-147.

Gorski, J.A., T. Talley, M. Qiu, L. Puelles, J.L. Rubenstein, and K.R. Jones. 2002. Cortical excitatory neurons and glia, but not GABAergic neurons, are produced in the Emx1expressing lineage. J Neurosci. 22:6309-6314.

Haglund, M.M., and P.A. Schwartzkroin. 1990. Role of Na-K pump potassium regulation and IPSPs in seizures and spreading depression in immature rabbit hippocampal slices. $J$ Neurophysiol. 63:225-239.

Heinemann, U., and H.D. Lux. 1977. Ceiling of stimulus induced rises in extracellular potassium concentration in the cerebral cortex of cat. Brain Res. 120:231-249.

Hermes, D., D.G.A. Kasteleijn-Nolst Trenite, and J. Winawer. 2017. Gamma oscillations and photosensitive epilepsy. Curr Biol. 27:R336-R338.

Hershko, A., and A. Ciechanover. 1998. The ubiquitin system. Annu Rev Biochem. 67:425479.

Higashi, K., A. Fujita, A. Inanobe, M. Tanemoto, K. Doi, T. Kubo, and Y. Kurachi. 2001. An inwardly rectifying $\mathrm{K}(+)$ channel, Kir4.1, expressed in astrocytes surrounds synapses and blood vessels in brain. Am J Physiol Cell Physiol. 281:C922-931.

Hsia, H.E., R. Kumar, R. Luca, M. Takeda, J. Courchet, J. Nakashima, S. Wu, S. Goebbels, W. An, B.J. Eickholt, F. Polleux, D. Rotin, H. Wu, M.J. Rossner, C. Bagni, J.S. Rhee, N. Brose, and H. Kawabe. 2014. Ubiquitin E3 ligase Nedd4-1 acts as a downstream 
target of PI3K/PTEN-mTORC1 signaling to promote neurite growth. Proc Natl Acad Sci U S A. 111:13205-13210.

Karwoski, C.J., H.K. Lu, and E.A. Newman. 1989. Spatial buffering of light-evoked potassium increases by retinal Muller (glial) cells. Science. 244:578-580.

Kawabe, H., A. Neeb, K. Dimova, S.M. Young, Jr., M. Takeda, S. Katsurabayashi, M. Mitkovski, O.A. Malakhova, D.E. Zhang, M. Umikawa, K. Kariya, S. Goebbels, K.A. Nave, C. Rosenmund, O. Jahn, J. Rhee, and N. Brose. 2010. Regulation of Rap2A by the ubiquitin ligase Nedd4-1 controls neurite development. Neuron. 65:358-372.

Kelley, K.W., L. Ben Haim, L. Schirmer, G.E. Tyzack, M. Tolman, J.G. Miller, H.H. Tsai, S.M. Chang, A.V. Molofsky, Y. Yang, R. Patani, A. Lakatos, E.M. Ullian, and D.H. Rowitch. 2018. Kir4.1-Dependent Astrocyte-Fast Motor Neuron Interactions Are Required for Peak Strength. Neuron. 98:306-319.

Kimura, T., H. Kawabe, C. Jiang, W. Zhang, Y.Y. Xiang, C. Lu, M.W. Salter, N. Brose, W.Y. Lu, and D. Rotin. 2011. Deletion of the ubiquitin ligase Nedd4L in lung epithelia causes cystic fibrosis-like disease. Proc Natl Acad Sci U S A. 108:32163221.

Larson, V.A., Y. Mironova, K.G. Vanderpool, A. Waisman, J.E. Rash, A. Agarwal, and D.E. Bergles. 2018. Oligodendrocytes control potassium accumulation in white matter and seizure susceptibility. Elife. 7.

LeBeau, F.E., S.K. Towers, R.D. Traub, M.A. Whittington, and E.H. Buhl. 2002. Fast network oscillations induced by potassium transients in the rat hippocampus in vitro. J Physiol. 542:167-179.

Livak, K.J., and T.D. Schmittgen. 2001. Analysis of relative gene expression data using realtime quantitative PCR and the 2(-Delta Delta C(T)) Method. Methods. 25:402-408.

Macia, E., M. Ehrlich, R. Massol, E. Boucrot, C. Brunner, and T. Kirchhausen. 2006. Dynasore, a cell-permeable inhibitor of dynamin. Dev Cell. 10:839-850.

Madisen, L., T.A. Zwingman, S.M. Sunkin, S.W. Oh, H.A. Zariwala, H. Gu, L.L. Ng, R.D. Palmiter, M.J. Hawrylycz, A.R. Jones, E.S. Lein, and H. Zeng. 2010. A robust and high-throughput Cre reporting and characterization system for the whole mouse brain. Nat Neurosci. 13:133-140.

Malagie, I., A.C. Trillat, C. Jacquot, and A.M. Gardier. 1995. Effects of acute fluoxetine on extracellular serotonin levels in the raphe: an in vivo microdialysis study. Eur $J$ Pharmacol. 286:213-217.

Maspero, E., E. Valentini, S. Mari, V. Cecatiello, P. Soffientini, S. Pasqualato, and S. Polo. 2013. Structure of a ubiquitin-loaded HECT ligase reveals the molecular basis for catalytic priming. Nat Struct Mol Biol. 20:696-701.

Minegishi, S., T. Ishigami, T. Kino, L. Chen, R. Nakashima-Sasaki, N. Araki, K. Yatsu, M. Fujita, and S. Umemura. 2016. An isoform of Nedd4-2 is critically involved in the renal adaptation to high salt intake in mice. Sci Rep. 6:27137.

Mizoguchi, A., T. Ueda, K. Ikeda, H. Shiku, H. Mizoguti, and Y. Takai. 1989. Localization and subcellular distribution of cellular ras gene products in rat brain. Brain Res Mol Brain Res. 5:31-44.

Nimmerjahn, A., F. Kirchhoff, J.N. Kerr, and F. Helmchen. 2004. Sulforhodamine 101 as a specific marker of astroglia in the neocortex in vivo. Nat Methods. 1:31-37.

Nolan, T., R.E. Hands, and S.A. Bustin. 2006. Quantification of mRNA using real-time RTPCR. Nat Protoc. 1:1559-1582.

Ohno, Y., H. Hibino, C. Lossin, A. Inanobe, and Y. Kurachi. 2007. Inhibition of astroglial Kir4.1 channels by selective serotonin reuptake inhibitors. Brain Res. 1178:44-51.

Pannasch, U., L. Vargova, J. Reingruber, P. Ezan, D. Holcman, C. Giaume, E. Sykova, and N. Rouach. 2011. Astroglial networks scale synaptic activity and plasticity. Proc Natl Acad Sci U S A. 108:8467-8472. 
Parra, J., S.N. Kalitzin, J. Iriarte, W. Blanes, D.N. Velis, and F.H. Lopes da Silva. 2003. Gamma-band phase clustering and photosensitivity: is there an underlying mechanism common to photosensitive epilepsy and visual perception? Brain. 126:1164-1172.

Perry, G., L.M. Brindley, S.D. Muthukumaraswamy, K.D. Singh, and K. Hamandi. 2014. Evidence for increased visual gamma responses in photosensitive epilepsy. Epilepsy Res. 108:1076-1086.

Persaud, A., P. Alberts, E.M. Amsen, X. Xiong, J. Wasmuth, Z. Saadon, C. Fladd, J. Parkinson, and D. Rotin. 2009. Comparison of substrate specificity of the ubiquitin ligases Nedd4 and Nedd4-2 using proteome arrays. Mol Syst Biol. 5:333.

Piper, R.C., I. Dikic, and G.L. Lukacs. 2014. Ubiquitin-dependent sorting in endocytosis. Cold Spring Harb Perspect Biol. 6.

Ren, L., M.T. Kucewicz, J. Cimbalnik, J.Y. Matsumoto, B.H. Brinkmann, W. Hu, W.R. Marsh, F.B. Meyer, S.M. Stead, and G.A. Worrell. 2015. Gamma oscillations precede interictal epileptiform spikes in the seizure onset zone. Neurology. 84:602-608.

Ripamonti, S., M.C. Ambrozkiewicz, F. Guzzi, M. Gravati, G. Biella, I. Bormuth, M. Hammer, L.P. Tuffy, A. Sigler, H. Kawabe, K. Nishimori, M. Toselli, N. Brose, M. Parenti, and J. Rhee. 2017. Transient oxytocin signaling primes the development and function of excitatory hippocampal neurons. Elife. 6.

Ronzaud, C., D. Loffing-Cueni, P. Hausel, A. Debonneville, S.R. Malsure, N. Fowler-Jaeger, N.A. Boase, R. Perrier, M. Maillard, B. Yang, J.B. Stokes, R. Koesters, S. Kumar, E. Hummler, J. Loffing, and O. Staub. 2013. Renal tubular NEDD4-2 deficiency causes NCC-mediated salt-dependent hypertension. J Clin Invest. 123:657-665.

Ross, P.L., Y.N. Huang, J.N. Marchese, B. Williamson, K. Parker, S. Hattan, N. Khainovski, S. Pillai, S. Dey, S. Daniels, S. Purkayastha, P. Juhasz, S. Martin, M. Bartlet-Jones, F. He, A. Jacobson, and D.J. Pappin. 2004. Multiplexed protein quantitation in Saccharomyces cerevisiae using amine-reactive isobaric tagging reagents. Mol Cell Proteomics. 3:1154-1169.

Sakatani, S., A. Seto-Ohshima, Y. Shinohara, Y. Yamamoto, H. Yamamoto, S. Itohara, and H. Hirase. 2008. Neural-activity-dependent release of S100B from astrocytes enhances kainate-induced gamma oscillations in vivo. J Neurosci. 28:10928-10936.

Schirmer, L., W. Mobius, C. Zhao, A. Cruz-Herranz, L. Ben Haim, C. Cordano, L.R. Shiow, K.W. Kelley, B. Sadowski, G. Timmons, A.K. Probstel, J.N. Wright, J.H. Sin, M. Devereux, D.E. Morrison, S.M. Chang, K. Sabeur, A. Green, K.A. Nave, R.J. Franklin, and D.H. Rowitch. 2018. Oligodendrocyte-encoded Kir4.1 function is required for axonal integrity. Elife. 7:e36428

Schmidt, C., D. Hesse, M. Raabe, H. Urlaub, and O. Jahn. 2013. An automated in-gel digestion/iTRAQ-labeling workflow for robust quantification of gel-separated proteins. Proteomics. 13:1417-1422.

Scholl, U.I., M. Choi, T. Liu, V.T. Ramaekers, M.G. Hausler, J. Grimmer, S.W. Tobe, A. Farhi, C. Nelson-Williams, and R.P. Lifton. 2009. Seizures, sensorineural deafness, ataxia, mental retardation, and electrolyte imbalance (SeSAME syndrome) caused by mutations in KCNJ10. Proc Natl Acad Sci U S A. 106:5842-5847.

Staub, O., S. Dho, P. Henry, J. Correa, T. Ishikawa, J. McGlade, and D. Rotin. 1996. WW domains of Nedd4 bind to the proline-rich PY motifs in the epithelial $\mathrm{Na}+$ channel deleted in Liddle's syndrome. EMBO J. 15:2371-2380.

Staub, O., and D. Rotin. 2006. Role of ubiquitylation in cellular membrane transport. Physiol Rev. 86:669-707.

Takumi, T., T. Ishii, Y. Horio, K. Morishige, N. Takahashi, M. Yamada, T. Yamashita, H. Kiyama, K. Sohmiya, S. Nakanishi, and et al. 1995. A novel ATP-dependent inward rectifier potassium channel expressed predominantly in glial cells. $J$ Biol Chem. 270:16339-16346. 
Tamura, H., L. Schild, N. Enomoto, N. Matsui, F. Marumo, and B.C. Rossier. 1996. Liddle disease caused by a missense mutation of beta subunit of the epithelial sodium channel gene. J Clin Invest. 97:1780-1784.

Tyanova, S., T. Temu, P. Sinitcyn, A. Carlson, M.Y. Hein, T. Geiger, M. Mann, and J. Cox. 2016. The Perseus computational platform for comprehensive analysis of (prote)omics data. Nat Methods. 13:731-740.

Vanli-Yavuz, E.N., O. Ozdemir, A. Demirkan, S. Catal, N. Bebek, U. Ozbek, and B. Baykan. 2015. Investigation of the possible association of NEDD4-2 (NEDD4L) gene with idiopathic photosensitive epilepsy. Acta Neurol Belg. 115:241-245.

Wallraff, A., R. Kohling, U. Heinemann, M. Theis, K. Willecke, and C. Steinhauser. 2006. The impact of astrocytic gap junctional coupling on potassium buffering in the hippocampus. $J$ Neurosci. 26:5438-5447.

Wang, M.X., X.T. Su, P. Wu, Z.X. Gao, W.H. Wang, O. Staub, and D.H. Lin. 2018. Kir5.1 regulates Nedd4-2-mediated ubiquitination of Kir4.1 in distal nephron. Am J Physiol Renal Physiol. 315:F986-F996.

Winchenbach, J., T. Duking, S.A. Berghoff, S.K. Stumpf, S. Hulsmann, K.A. Nave, and G. Saher. 2016. Inducible targeting of CNS astrocytes in Aldh111-CreERT2 BAC transgenic mice. F1000Res. 5:2934.

Woelk, T., B. Oldrini, E. Maspero, S. Confalonieri, E. Cavallaro, P.P. Di Fiore, and S. Polo. 2006. Molecular mechanisms of coupled monoubiquitination. Nat Cell Biol. 8:12461254.

Wojtowicz, A.M., L. van den Boom, A. Chakrabarty, N. Maggio, R.U. Haq, C.J. Behrens, and U. Heinemann. 2009. Monoamines block kainate- and carbachol-induced gammaoscillations but augment stimulus-induced gamma-oscillations in rat hippocampus in vitro. Hippocampus. 19:273-288.

Zamanillo, D., R. Sprengel, O. Hvalby, V. Jensen, N. Burnashev, A. Rozov, K.M. Kaiser, H.J. Koster, T. Borchardt, P. Worley, J. Lubke, M. Frotscher, P.H. Kelly, B. Sommer, P. Andersen, P.H. Seeburg, and B. Sakmann. 1999. Importance of AMPA receptors for hippocampal synaptic plasticity but not for spatial learning. Science. 284:18051811.

Zhou, R., S.V. Patel, and P.M. Snyder. 2007. Nedd4-2 catalyzes ubiquitination and degradation of cell surface ENaC. J Biol Chem. 282:20207-20212.

Zhu, J., K.Y. Lee, K.A. Jewett, H.Y. Man, H.J. Chung, and N.P. Tsai. 2017. Epilepsyassociated gene Nedd4-2 mediates neuronal activity and seizure susceptibility through AMPA receptors. PLoS Genet. 13:e1006634. 


\section{LIST OF ABBREVIATION}

Nedd4, Neuronal precursor expressed developmentally downregulated 4; Ubi, ubiquitin;

Kir4.1, inwardly rectifying $\mathrm{K}^{+}$channel 4.1; KO, knockout; Cx43, connexin43; Cx30, connexin30; E1, ubiquitin activating enzyme; E2, ubiquitin conjugating enzyme; E3, ubiquitin ligase; HECT, homologous to the E6AP carboxyl terminus; N4-2, Nedd4-2; N4-1, Nedd4-1; LFP, local field potential; N4-1/2 bDKO, brain-specific conditional Nedd41/Nedd4-2 knockout; N4-2 bKO, brain-specific Nedd4-2 knockout; SM, synaptic plasma membrane fraction; iTRAQ, isobaric tags for relative and absolute quantitation; N4-1 bKO, brain-specific Nedd4-1 single knockout; CTL, control; Kir4.1-HA, HA-tagged Kir4.1; Cx43HA, HA-tagged connexin43; K63-Ub-chains, K63-linked polyubiquitin chain; K48-Ubchains, K48-linked polyubiquitin chain; ERAD, endoplasmic reticulum-associated degradation; WT, wild type; C/S, catalytically inactive point mutant; AM, acetomethoxy; FRAP, fluorescence recovery after photobleaching; tdTom, tdTomato; N4-2 AstKO, astrocyte-specific Nedd4-2 knockout; PV, parvalbumin; ACSF, artificial cerebrospinal fluid 


\section{FIGURES AND LEGENDS}

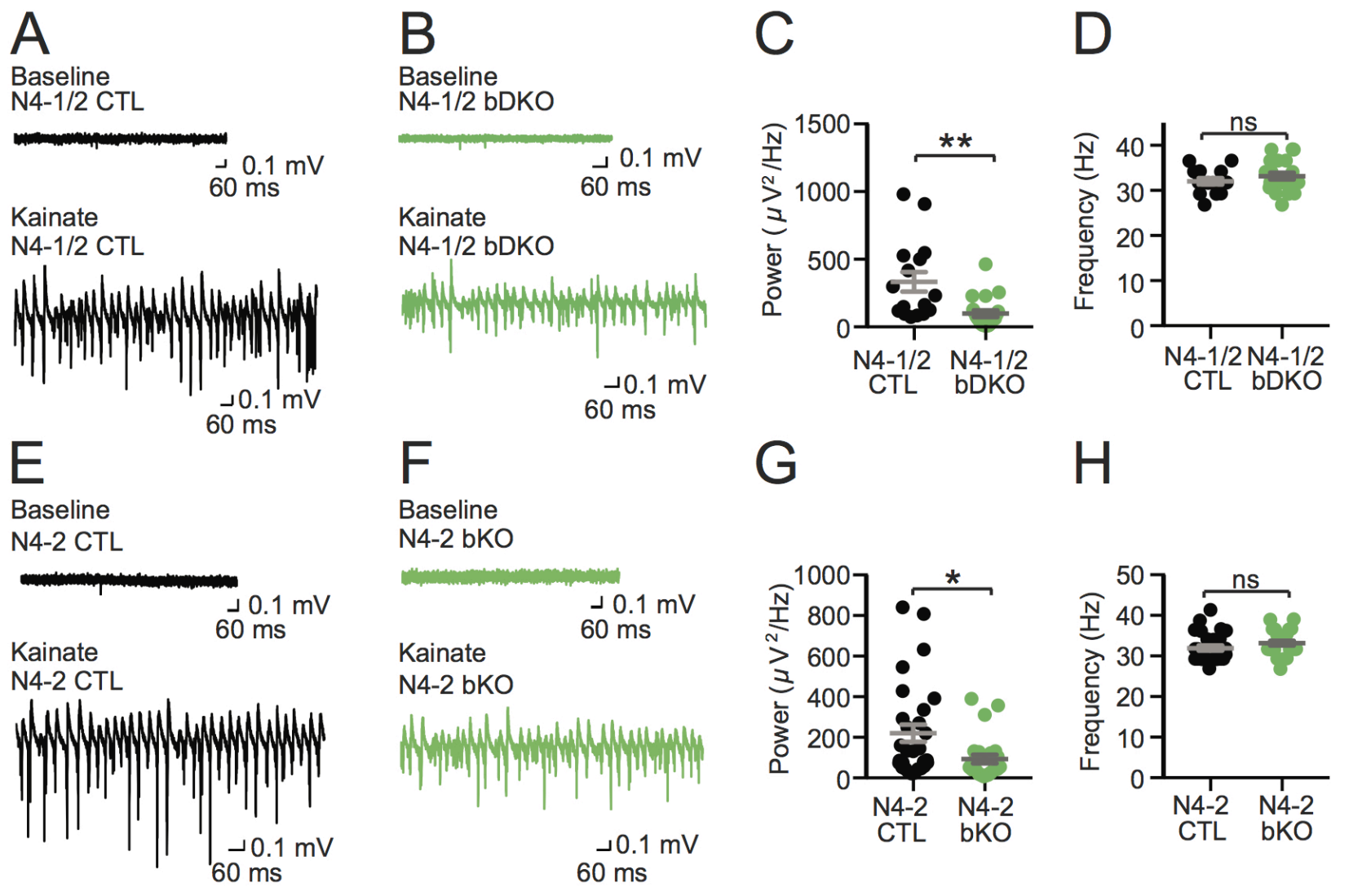

Figure 1. $\gamma$-Oscillations in acute slices from Nedd4 brain-specific knockout mice. (A to D) Reduced power of $\gamma$-oscillations in N4-1/2 bDKO mice. Representative recordings in CA3 hippocampal regions of acute brain slices from N4-1/2 CTL (A) and N4-1/2 bDKO (B) mice before (Baseline) and during (Kainate) induction of $\gamma$-oscillations with $100 \mathrm{nM}$ kainate. Average powers (C) and frequencies (D) of $\gamma$-oscillations in N4-1/2 CTL (black dots) and N4-1/2 bDKO (green dots). (E to H) Reduced power of $\gamma$-oscillations in N4-2 bKO mice. Representative recordings in CA3 region of hippocampus slices from N4-2 CTL (E) and N4$2 \mathrm{bKO}$ (F) mice before (Baseline) and during (Kainate) the application of $100 \mathrm{nM}$ kainate. Average powers (G) and frequencies (H) of $\gamma$-oscillations in N4-2 CTL (black dots) and N42 bKO (green dots). Numbers of recorded slices (n); (C) and (D), $n=19$ for N4-1/2 CTL, $\mathrm{n}=21$ for N4-1/2 bDKO; $(\mathbf{G})$ and $\mathbf{( H )}, \mathrm{n}=29$ for N4-2 CTL, $\mathrm{n}=25$ for N4-2 bDKO. Results are shown as mean \pm SEM. ${ }^{* *}, 0.001<p<0.01 ;{ }^{*}, 0.01<p<0.05 ;$ ns, $0.05<p$ (Student's t-test). See also Table S2. 

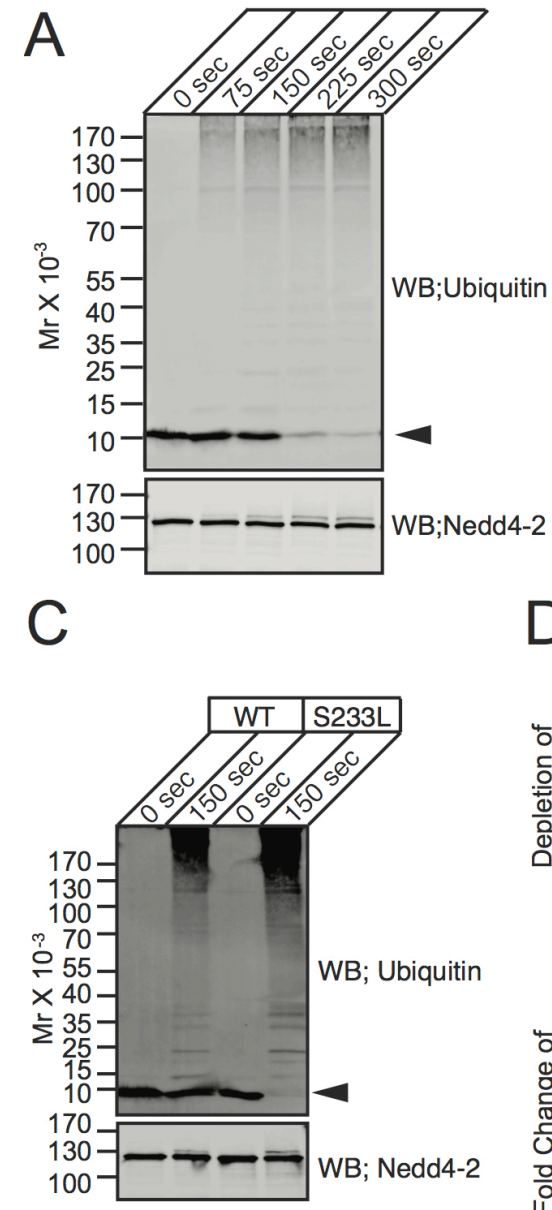

$\mathrm{B}$
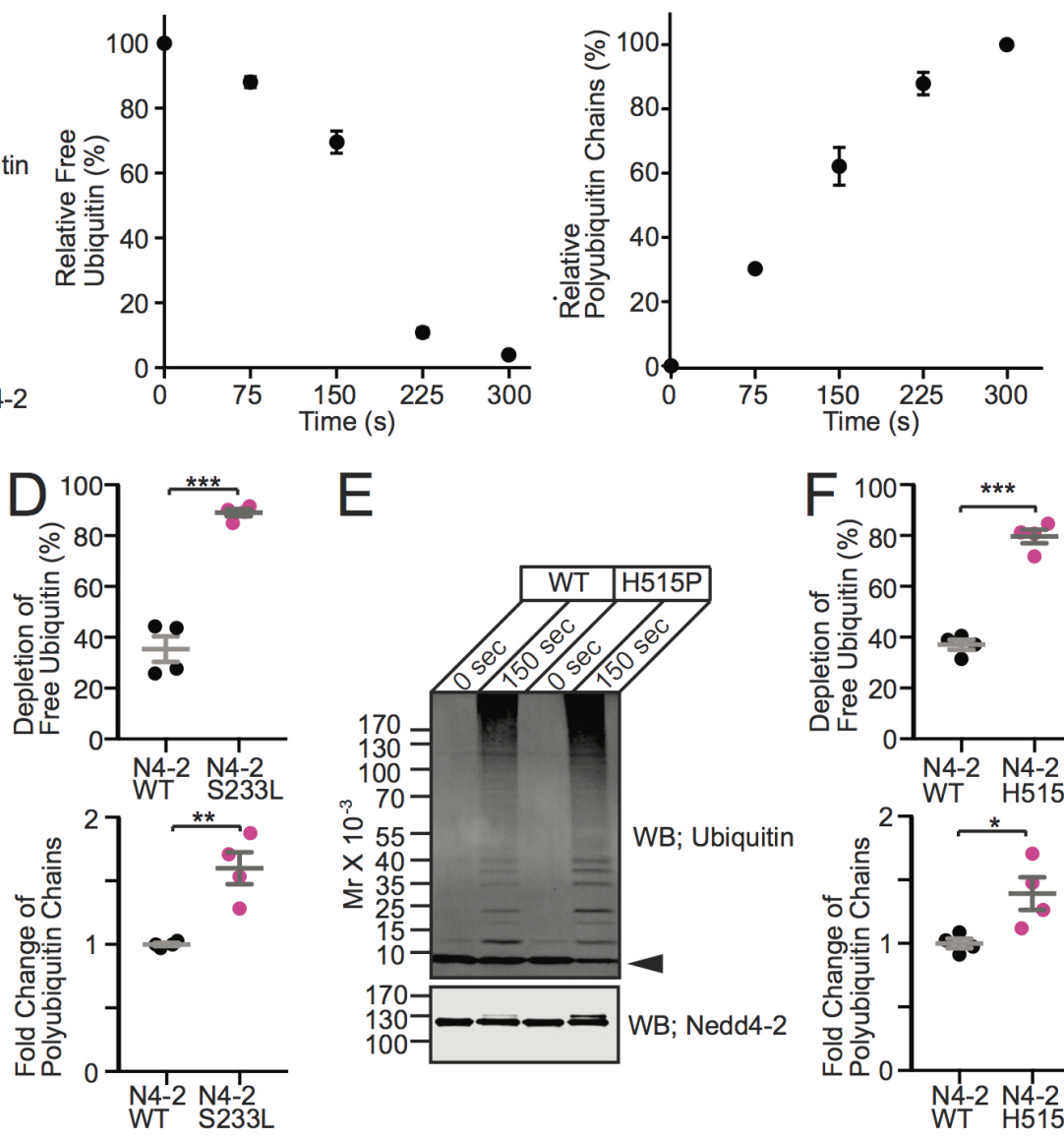
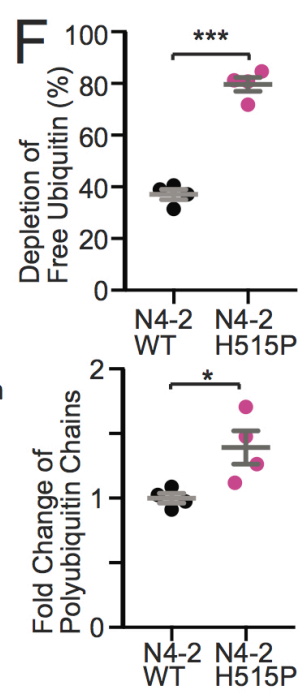

Figure 2. Polyubiquitin chain formation activities of Nedd4-2 WT, S233L, and H515P mutants. (A) Time course of ubiquitination using recombinant wild type Nedd4-2 (Nedd4-2 WT). Purified Ubi was incubated with ATP, E1, E2, and Nedd4-2 WT for indicated durations. Samples were subjected for Western blotting using anti-Ubi (top panel) and anti-Nedd4-2 (bottom panel) antibodies. Note the time-dependent polyUbi chain formation at the expense of free Ubi (arrowhead). (B) The average time course of the depletion of free Ubi (left) and formation of polyUbi chains (right) in in vitro ubiquitination assay in $(\mathbf{A})(\mathrm{n}=3$ replicates). (C to F) S233L (C and D) and H515P (E and F) missense point mutants of Nedd4-2 cause gain-of-function of the catalytic activity. (C and $\mathbf{E})$ Representative images of Western blotting using anti-Ubi (upper panel) and anti-Nedd4-2 (lower panel) antibodies for in vitro ubiquitination assay samples using Nedd4-2 WT and mutants [S233L in (C) and H515P in (E)]. Arrowheads, free Ubi. (D and F) Quantifications of depletion of free Ubi (top dot plots) and formation of polyUbi chains relative to N4-2 WT (bottom dot plots) after $150 \mathrm{sec}$ incubation. Nedd4-2 WT (black dots) and Nedd4-2 mutants (magenta dots). Results are shown as mean \pm SEM. Numbers of experiments are 4 for each set of experiments. ***, $p<0.001 ; * *, 0.001<p<0.01 ; *, 0.01<p<0.05$ (Student's t-test). See also Table S2. 
A

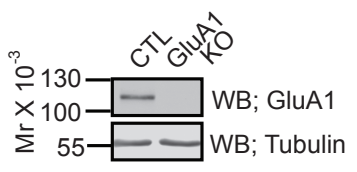

B

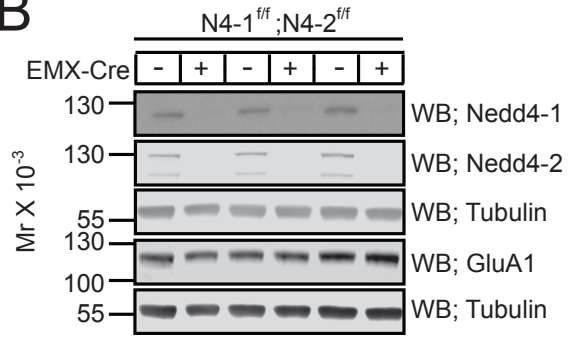

C

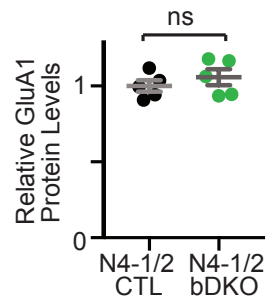

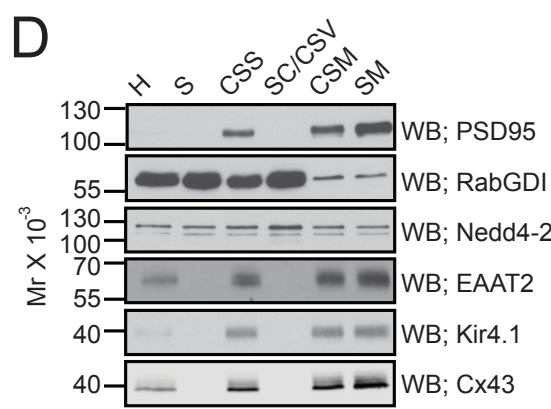

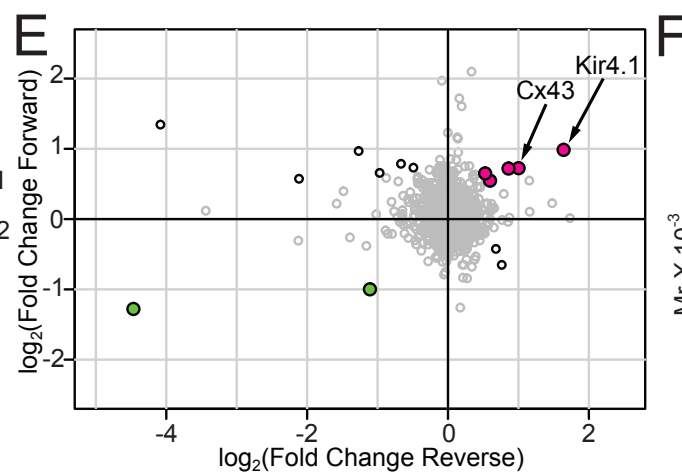

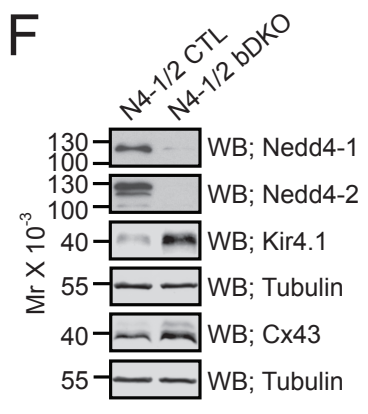
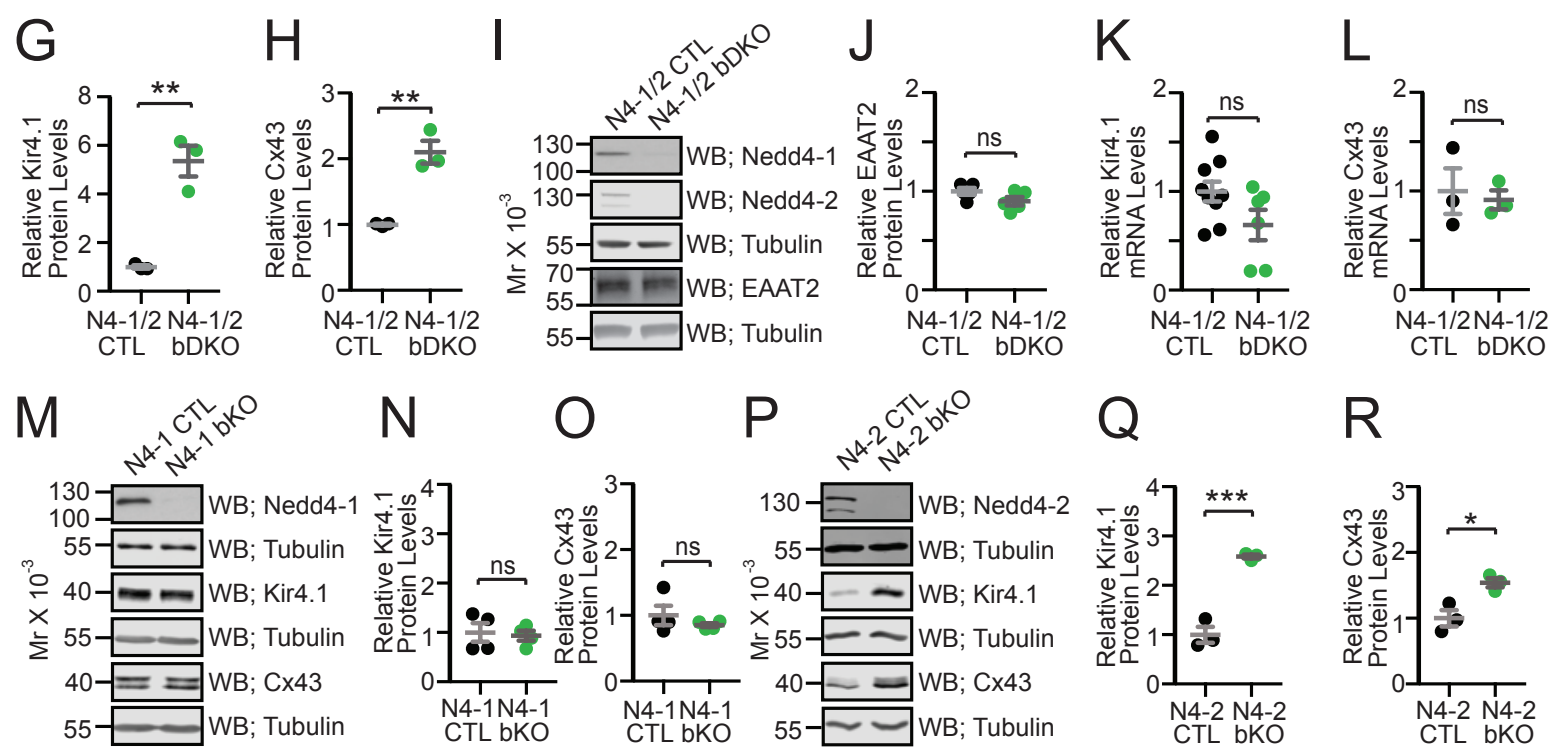

Figure 3. Screening of proteins upregulated in Nedd4-1/2 brain-specific knockout mice.

(A) Specificity of anti-GluA1 antibody verified with cortical homogenates from CTL and GluA1 KO. (B and C) Quantitative Western blots with the anti-GluA1 antibody using cortical homogenates from N4-1/2 CTL and N4-1/2 bDKO mice. In (C), black dots, N4-1/2 CTL; green dots, N4-1/2 bDKO. (D) Protein profiles of PSD95, RabGDI, Nedd4-2, EAAT2, Kir4.1, and $\mathrm{Cx} 43$ in subcellular fractionated samples from wild type mice. H, Homogenate; S, Soluble; CSS, Crude Synaptosome; SC/CSV, Synaptic Cytoplasm/Crude Synaptic Vesicle; CSM, Crude Synaptic Membrane; and SM, Pure Synaptic Membrane Fractions. (E) Scatter plot of relative protein abundance as quantified by mass spectrometry. The log2-transformed fold-change ratios between N4-1/2 bDKO and N4-1/2 CTL in the forward (y-axis) against reverse (x-axis) experiments were plotted. Black circles indicate proteins significantly changed in both experiments (Significance B values $<0.05$ ). Color filling indicates proteins consistently up-regulated (magenta) or down-regulated (green) in the same direction in both experiments. (F) Representative Western blotting of cortical brain lysates from N4-1/2 CTL and N4-1/2 bDKO mice. (G and H) Levels of Kir4.1 (G) and Cx43 (H) in N4-1/2 CTL 
(black dots) and N4-1/2 bDKO (green dots). (I and J) Quantitative Western blotting using the anti-EAAT2 antibody showed no difference between N4-1/2 CTL (black dots) and N41/2 bDKO (green dots). (K and L) Kir4.1 (K) and Cx43 (L) mRNA levels were not significantly different in N4-1/2 CTL (black dots) and N4-1/2 bDKO (green dots). (M to R) Protein levels of Kir4.1 and Cx43 in N4-1 bKO (M to O) and N4-2 bKO (P to R) cortical lysates. Results are shown as mean \pm SEM. Numbers of mice $(n) ;(\mathbf{C}), n=5$ for each genotype; (G) and (H), n=3 for each genotype; $(\mathbf{J}), n=5$ for each genotype; $(\mathbf{K}), \mathrm{n}=9$ for N41/2 CTL, $n=6$ for N4-1/2 bDKO; (L), n=3 for each genotype; (N) and (O), $n=4$ for each genotype; (Q) and (R), $\mathrm{n}=3$ for each genotype. ***, $p<0.001$; **, $0.001<p<0.01$; *, $0.01<p<0.05 ;$ ns, $0.05<p$. Student's t-test. See also Tables S1 and S2. 
bioRxiv preprint doi: https://doi.org/10.1101/683441; this version posted July 4, 2019. The copyright holder for this preprint (which was not certified by peer review) is the author/funder. All rights reserved. No reuse allowed without permission.

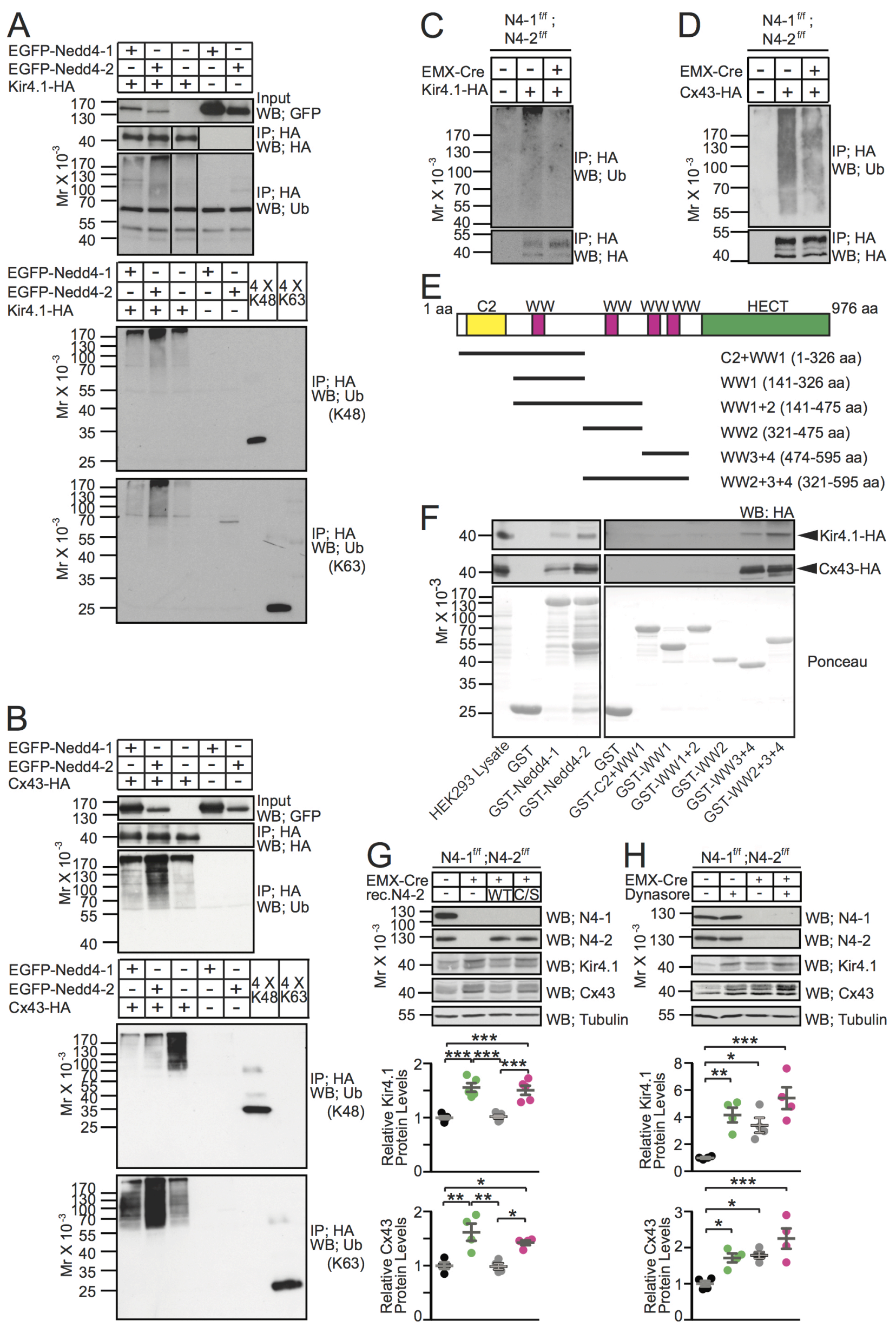


Figure 4. Biochemical characterization of Kir4.1 and $\mathrm{Cx} 43$ as substrates of Nedd4-2. (A and B) Kir4.1-HA (A) or Cx43-HA (B) was expressed in HEK293FT cells in the presence or absence of EGFP-tagged Nedd4 E3s. Levels of Nedd4 E3s were studied by an anti-GFP antibody (top panels). HA-tagged substrates were immunoprecipitated (IP) with an anti-HA antibody and subjected for Western blotting using anti-HA (second panel) and anti-Ubi (third panel) antibodies. Note increased smear signals cross-reacting the anti-Ubi antibody when EGFP-Nedd4-1 (first lane) or EGFP-Nedd4-2 (second lane) were co-expressed. Lanes in the second and third panels in (A) were run on the same gel but were noncontiguous. Patterns of anti-Ubi Western blotting differs between (A) and (B) because different anti-Ubi antibodies were used. Same samples were blotted with anti-K48-linked and anti-K63-linked polyUbi chain antibodies (bottom two panels in (A) and (B)). Equal amounts of K48- and K63-linked tetra Ubi chains were loaded in right two lanes for SDS-PAGE together with ubiquitination assay samples. Note that signals from K48-linked and K63-linked tetra Ubi chains in the bottom two blots are comparable, indicating that the anti-K48 and anti-K63 antibodies have almost same titers. (C and D) N4-1/2 CTL (EMX-Cre -) and N4-1/2 bDKO (EMX-Cre +) astrocytes were infected with lentivirus expressing Kir4.1-HA (C) or Cx43-HA (D). Immunoprecipitated HA-tagged proteins were analyzed by Western blotting with anti-Ubi (upper panel) and anti-HA (lower panel) antibodies. Images are representative of at least two independent experiments. (E) Domain structure of Nedd4-2 (Accession number, NM_001114386). The amino acid sequences covered by truncated mutants of Nedd4-2 are indicated. (F) Affinity pull-down experiment using purified GST-tagged Nedd4 E3s with Kir4.1-HA (top panels) and Cx43-HA (middle panels) expressed in and extracted from HEK293FT cells. Immobilized GST-tagged proteins are stained with Ponceau (bottom panels). Approximately twice more Kir4.1-HA and Cx43-HA bound to GST-Nedd4-2 than to GST-Nedd4-1 (3rd and 4th lanes in the left top and the left middle panels) on the shown blots. Images are representative of at least two independent experiments. (G) Rescue of Kir4.1 (third Western blotting panel) and Cx43 (fourth Western blotting panel) levels in N4-1/2 bDKO astrocytes (EMX-Cre + ) over the control (EMX-Cre -) by re-expressing recombinant wild type Nedd4-2 (rec.N4-2 WT) but not by the inactive mutant of Nedd4-2 (rec.N4-2 C/S). In dot plots, black dots, control astrocytes; green dots, N4-1/2 bDKO astrocytes; gray dots, N4-1/2 bDKO astrocytes expressing recombinant rec.N4-2 WT; magenta dots, N4-1/2 bDKO astrocytes expressing rec.N4-2 C/S. (H) Upregulation of Kir4.1 (third Western blotting panel) and Cx43 (fourth Western blotting panel) by blocking endocytosis using dynasore in cultured N4-1/2 CTL astrocytes (EMX-Cre -) but not in N4-1/2 bDKO astrocytes (EMX-Cre + ). In dot plots, black dots, control astrocytes treated with vehicle; green dots, control astrocytes treated with $100 \mu \mathrm{M}$ dynasore; gray dots, N4-1/2 bDKO astrocytes treated with vehicle; magenta dots, N4-1/2 bDKO astrocytes treated with $100 \mu \mathrm{M}$ dynasore. Results are shown as mean \pm SEM. Numbers of mice $(n)$; the bot plot for Kir4.1 in (G), $n=5$ for each assay point; other plots, $\mathrm{n}=4$ for each assay point. ***, $p<0.001$; **, $0.001<p<0.01$; *, $0.01<p<0.05$; no asterisk, $0.05<p$ (One-way ANOVA with Tukey's post-hoc test). See also Tables S3 and S4. 

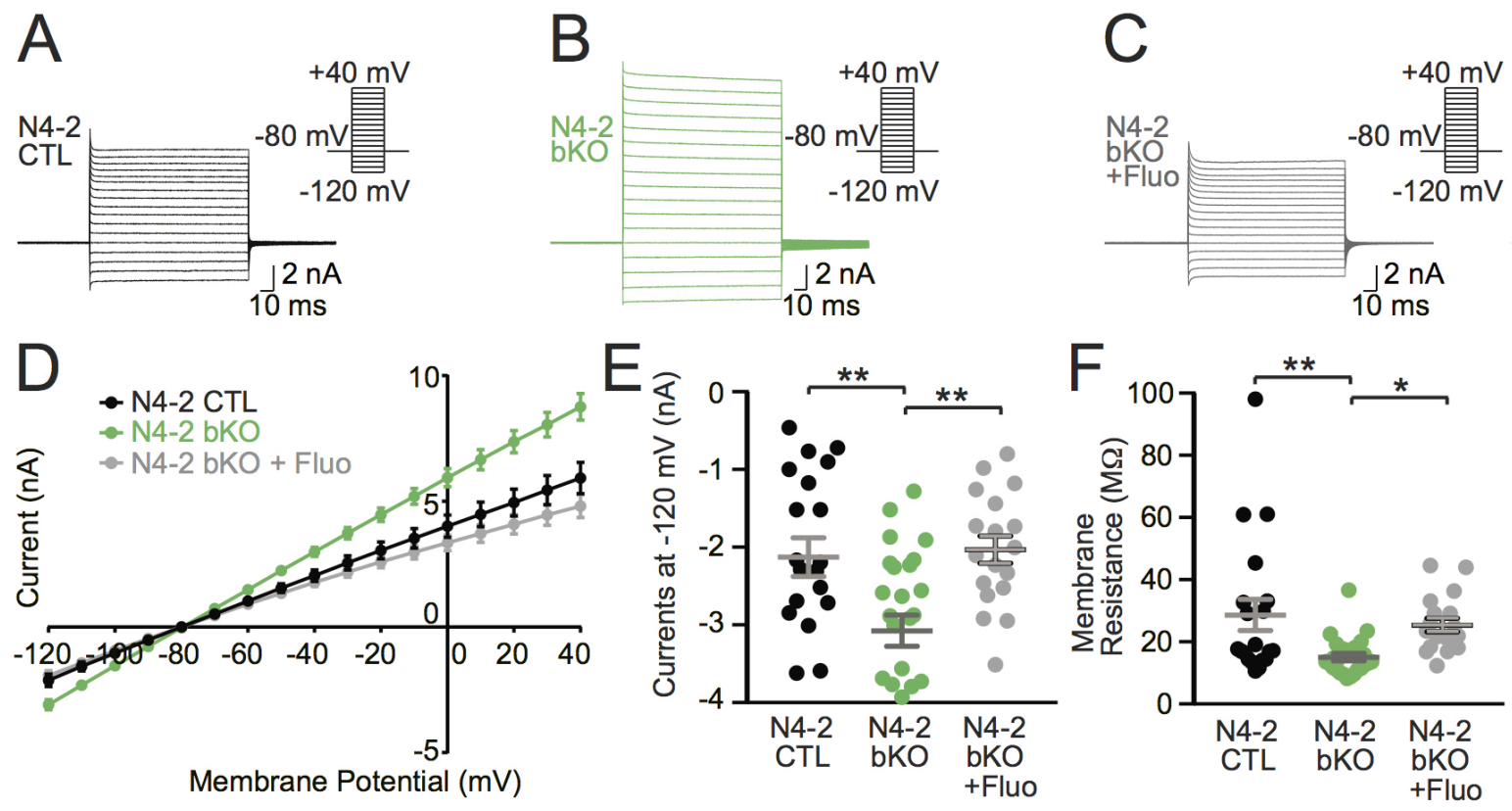

Figure 5. Kir4.1-dependent increase in membrane conductance in $\mathrm{N4-2}$ bKO astrocytes. (A to C) Example current traces in N4-2 CTL astrocytes (A), N4-2 bKO astrocytes (B), and N4-2 bKO astrocytes treated with fluoxetine (Fluo) (C). Cells were voltage clamped at -80 $\mathrm{mV}$ and $+10 \mathrm{mV}$ voltage steps were applied from $-120 \mathrm{mV}$ to $+40 \mathrm{mV}$ during recording currents. (D to F) Quantifications of rescues of defects in N4-2 bKO by fluoxetine. (D) Voltage-current plots from N4-2 CTL (black trace), N4-2 bKO (green trace), and N4-2 bKO treated with fluoxetine (gray trace). (E and F) The current $(\mathbf{E})$ and membrane resistance (F) at the membrane potential of $-120 \mathrm{mV}$ in N4-2 bKO (green dots) astrocytes were decreased as compared to N4-2 CTL astrocytes (black dots). Decreased currents (E) and membrane resistance (F) were restored to control levels by the application of Fluo in N4-2 bKO astrocytes (gray dots). Results are shown as mean \pm SEM. Numbers of recorded cells (n) in (D) to (F); n=20 for N4-2 CTL, $\mathrm{n}=26$ for N4-2 bKO, n=18 for N4-2 bKO + Fluo. **, $0.001<p<0.01 ; *, 0.01<p<0.05$; no asterisk, $0.05<p$. (One-way ANOVA with Tukey's posthoc test for $\mathbf{E}$ and $\mathbf{F}$ ). See also Tables S3 and S4. 

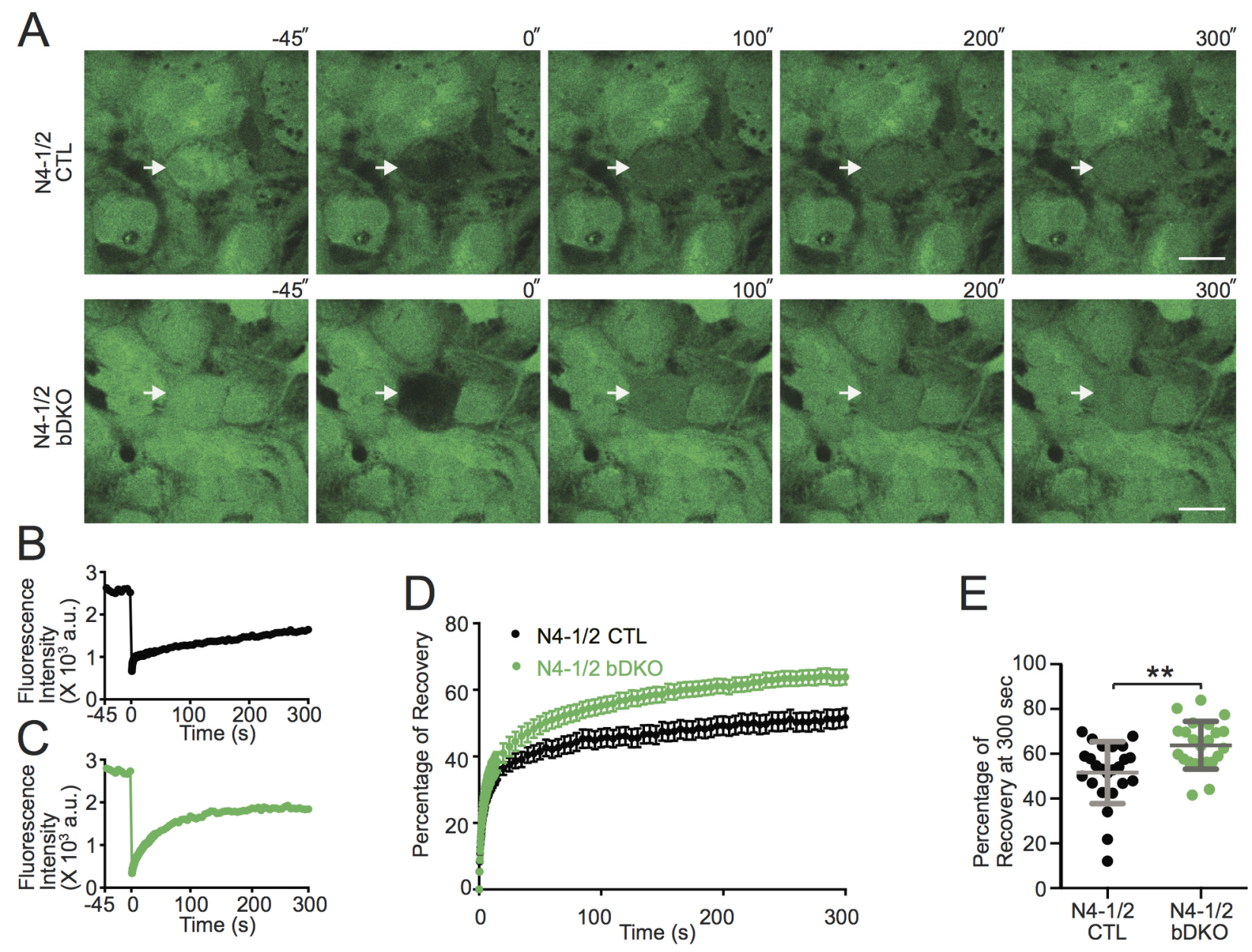

Figure 6. Augmented astrocyte coupling in the absence of Nedd4 E3s. (A) Representative images of FRAP live imaging experiments in N4-1/2 CTL (top panels) and N4-1/2 bDKO astrocytes (bottom panels). Primary astrocytes prepared from N4-1/2 CTL and N4-1/2 bDKO mice were loaded with calcein-AM. Calcein in the astrocytes indicated with arrows was bleached and FRAP was recorded. Scale bars, $20 \mu \mathrm{m}$. (B and C) Time courses of FRAP at bleached cells in (A) for N4-1/2 CTL (B) and N4-1/2 bDKO (C) astrocytes. (D) Average FRAP time courses of N4-1/2 CTL (black trace) and N4-1/2 bDKO (green trace) astrocytes. (E) Average fluorescence recoveries at $300 \mathrm{sec}$ after bleaching. N4-1/2 bDKO showed a significant increase in the recovery of calcein fluorescence over N4-1/2 CTL. Results are shown as mean \pm SEM. Numbers of imaged cells ( $n$ ); $n=24$ for N4-1/2 CTL, $n=23$ for N4-1/2 bDKO. ${ }^{* *}, 0.001<p<0.01$. (Student's t-test). See also Table S2. 


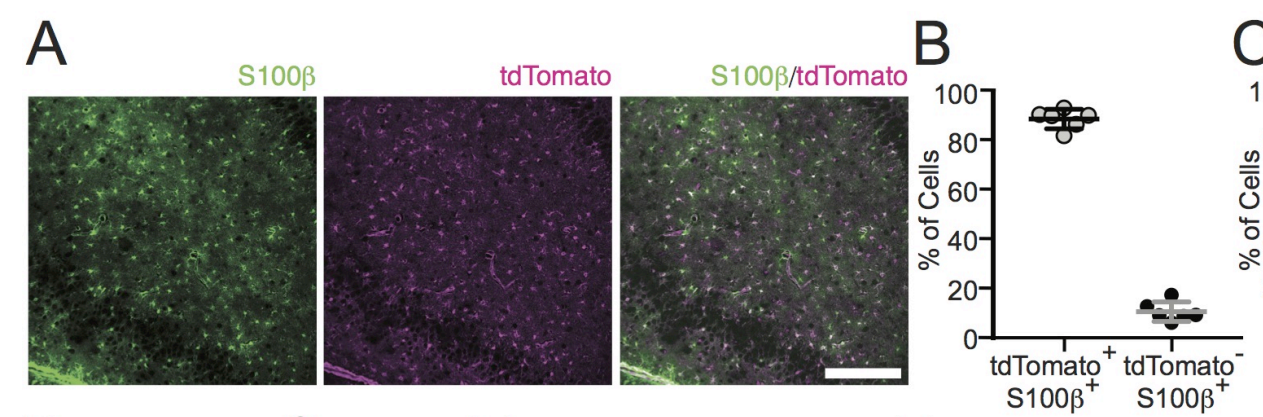

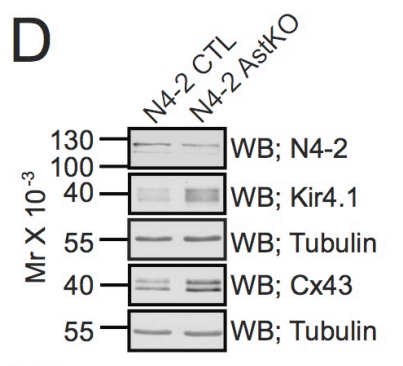
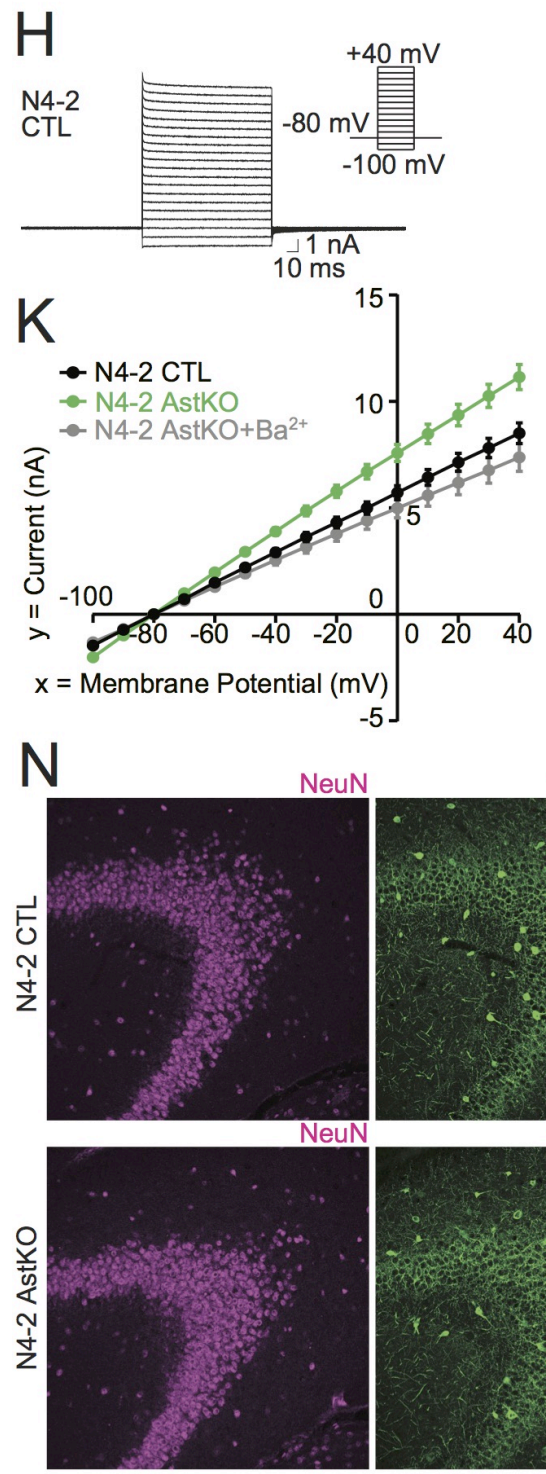
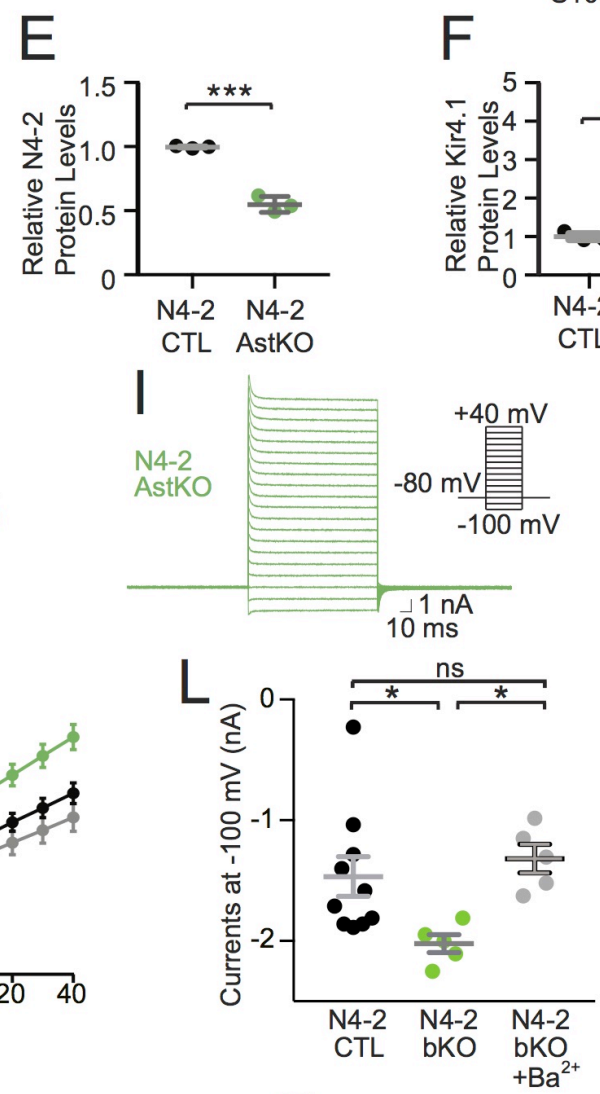

$\mathrm{O}$

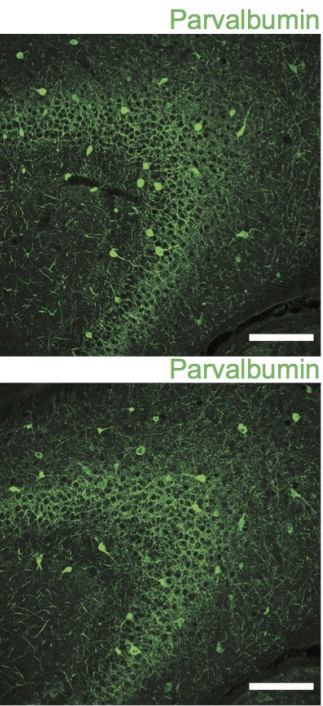

$\mathrm{F}$
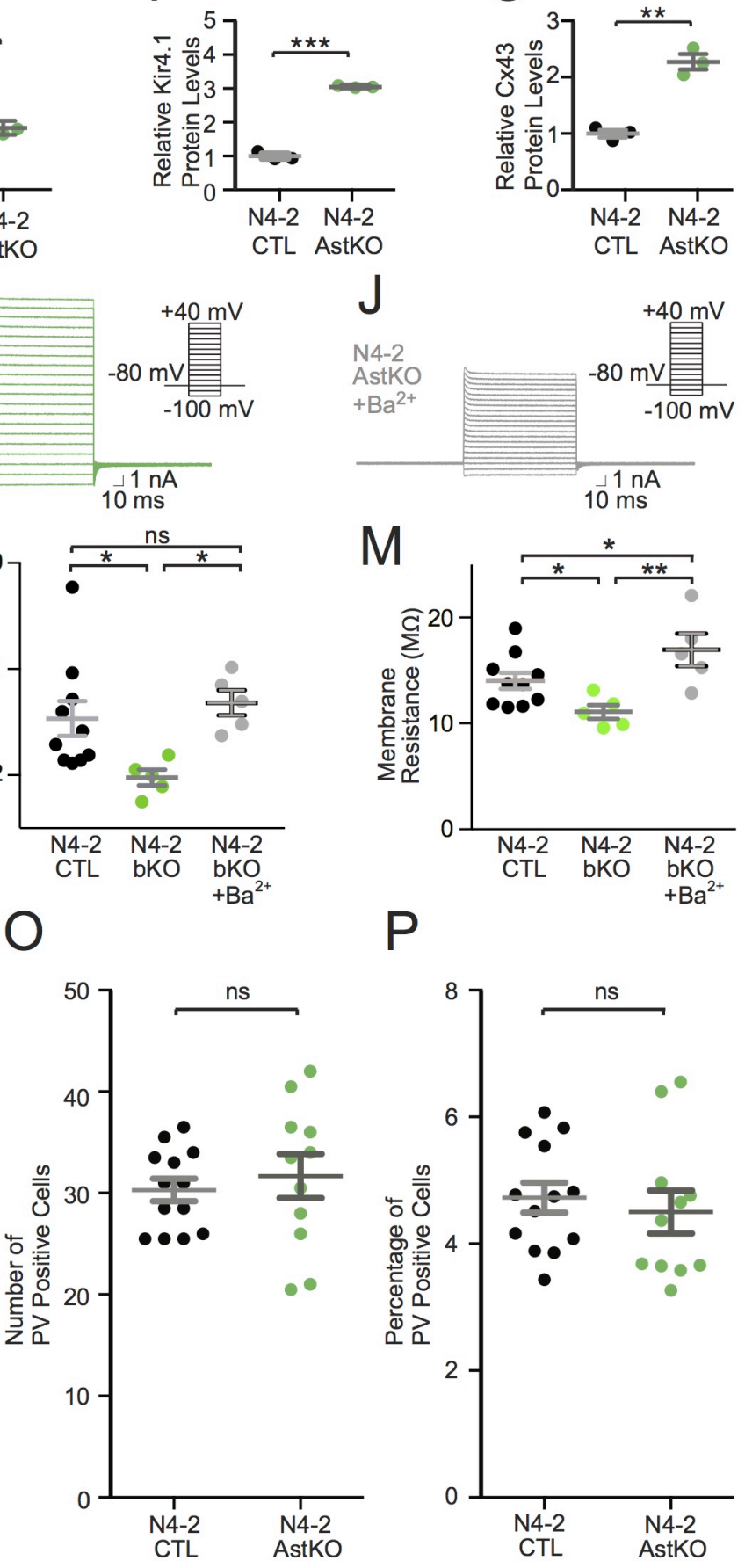

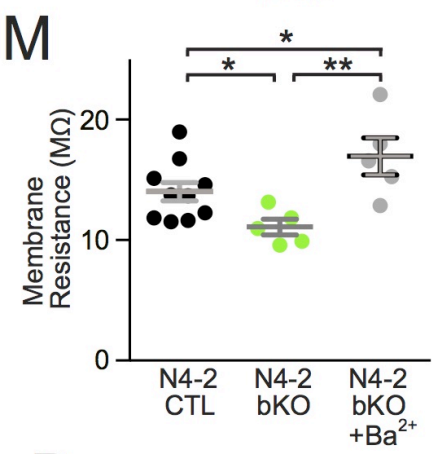

$\mathrm{P}$

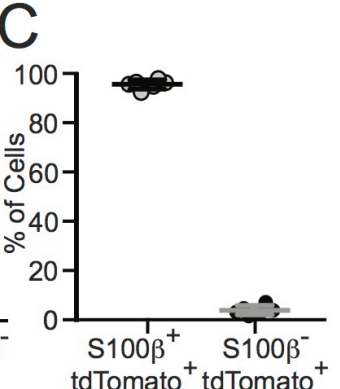

G
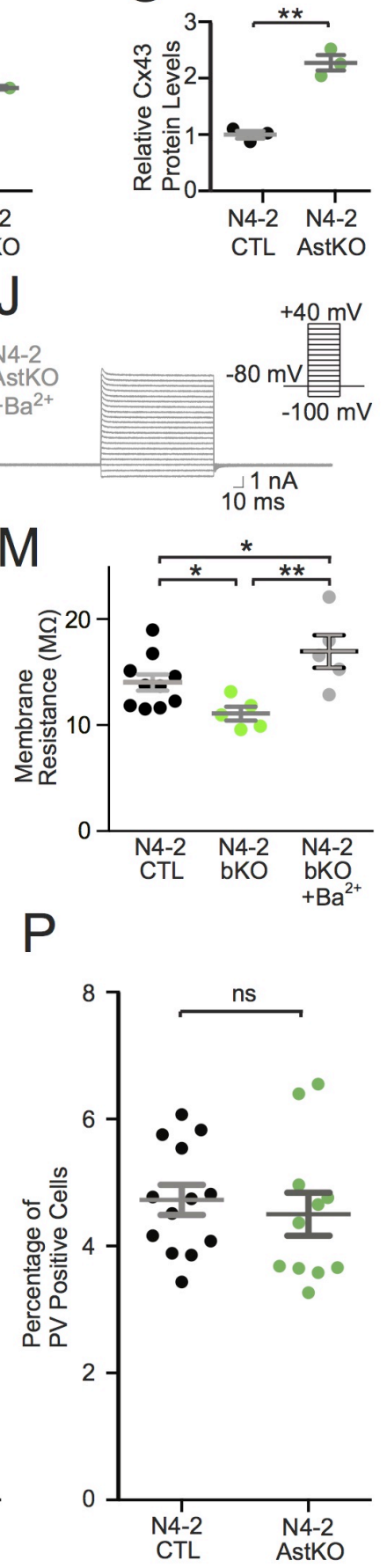

Figure 7. Characterization of astrocyte specific N4-2 conditional KO. (A) Aldh1l1CreERT2 mouse crossed with tdTomato-expressing Cre indicator mouse (Aldh1llCreERT2;ROSA26-tdTom) was injected with tamoxifen to induce Cre recombination with 
the same protocol used for Nedd4- $2^{\mathrm{f} / \mathrm{f}}$;Aldh1l1-CreERT2 (N4-2 AstKO) mice. Hippocampal CA3 region was immunostained for an astrocyte marker S100 $\beta$. (B) The efficiency of Cre recombination in astrocytes. $88.5 \pm 1.5$ (SEM) \% of S100 $\beta$-positive cells are also positive for tdTomato. (C) The specificity of Cre recombination. $95.1 \pm 0.77$ (SEM) \% of tdTomatoexpressing Cre-recombined cells are positive for S100 $\beta$. Total number of cells counted in (B) and (C) are 898 all together. 6 images taken from 3 mice were analyzed. (D) Representative Western blotting results using N4-2 CTL and N4-2 AstKO with antibodies to N4-2, Kir4.1, Tubulin, and Cx43. (E to G) Quantification of relative N4-2, Kir4.1, and Cx43 protein levels. (H to $\mathbf{M})$ An increase in plasma membrane currents in N4-2 AstKO were restored by blocking Kir4.1 with barium chloride $\left(\mathrm{Ba}^{2+}\right)$. Experiments were performed in a way similar to those in Figure 5. All mutants were crossed with ROSA26-tdTom. (H to J) Example trances of N4-2 CTL crossed with ROSA26-tdTom (H), N4-2 AstKO crossed with ROSA26-tdTom (I), and N4-2 AstKO crossed with ROSA26-tdTom and treated with $\mathrm{Ba}^{2+}$ (J). (K to M) Voltage-current plots $(\mathbf{K})$, currents at $-100 \mathrm{mV}(\mathbf{L})$, and membrane resistance $(\mathbf{M})$ from three conditions. ( $\mathbf{N}$ to $\mathbf{P})$ Unchanged number of parvalbumin-positive cells in N4-2 AstKO. (N) Representative images of N4-2 CTL (top panels) and N4-2 AstKO (bottom panels) hippocampal sections stained with anti-Neuronal Nuclei (NeuN) and anti-Parvalbumin (PV) antibodies. Scale bars, $300 \mu \mathrm{m}$. (O and P) Absolute numbers of PV-positive cells in each entire imaged field (O) and the percentages of PV positive cells with respect to total NeuNpositive cells (P). Numbers of mice for (E) to (G) are 3 for each genotype. Numbers of recorded cells $(\mathrm{n})$ in $(\mathbf{K})$ to $(\mathbf{M}) ; n=10$ for N4-2 CTL, $n=5$ for N4-2 AstKO, $n=5$ for N4-2 AstKO $+\mathrm{Ba}^{2+}$. Numbers of brain sections in $(\mathbf{O}$ and $\mathbf{P})(\mathrm{n}) ; \mathrm{n}=13$ for N4-2 CTL, $\mathrm{n}=11$ for N4-2 AstKO. ${ }^{* * *}, p<0.001 ; * *, 0.001<p<0.01 ; *, 0.01<p<0.05$; ns, $0.05<p$. (Student's t-test for $\mathbf{E}, \mathbf{F}, \mathbf{G}, \mathbf{O}$, and $\mathbf{P}$; One-way ANOVA with Newman-Keuls test for $\mathbf{L}$ and $\mathbf{M}$ ). See also Tables S2, S3, and S4. 


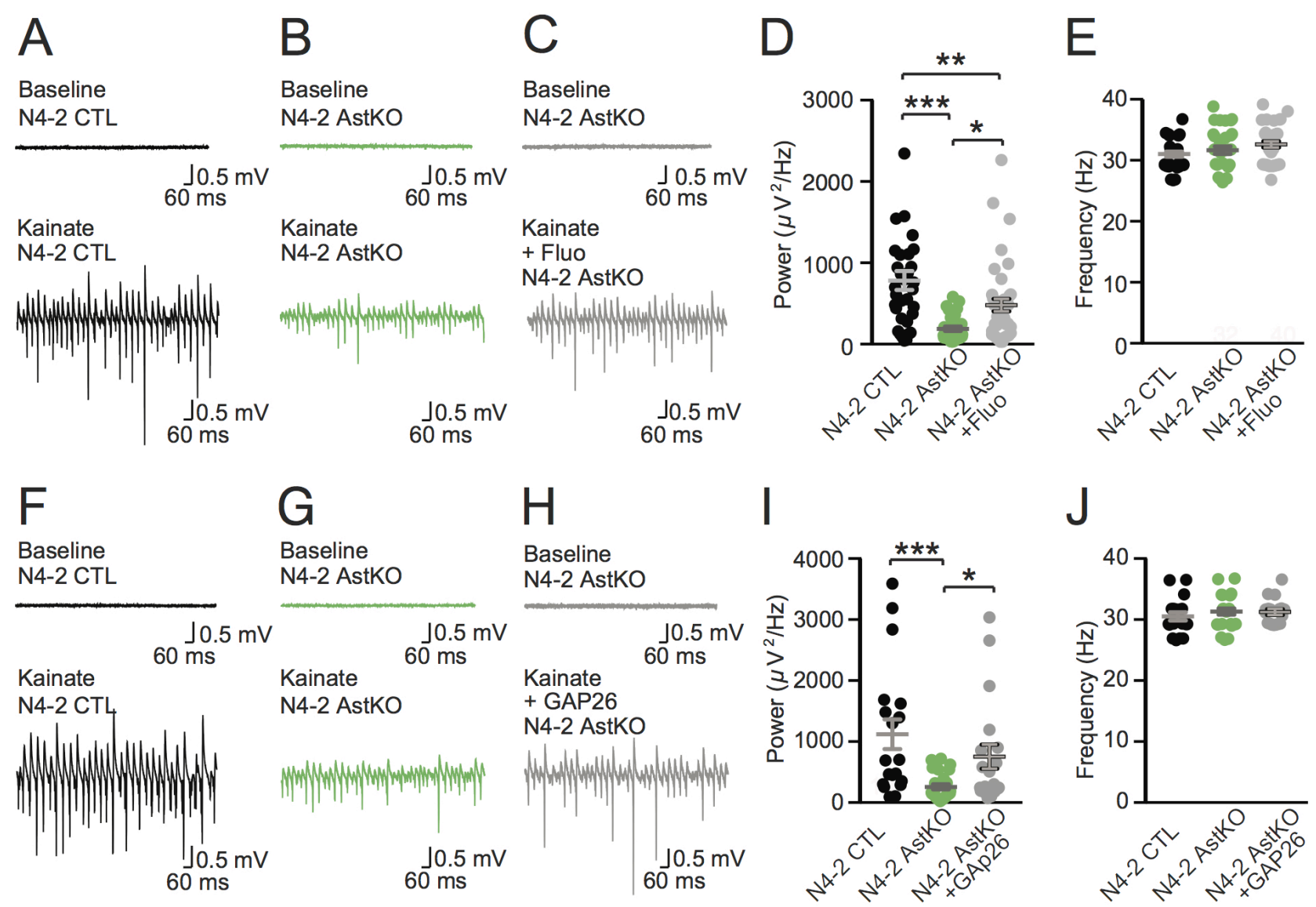

Figure 8. Rescue of reduced $\gamma$-oscillation in N4-2 AstKO by pharmacological inhibition of Kir4.1 or gap junction. (A and B) Representative recordings in the CA3 region of acute hippocampal slices from N4-2 CTL (A) and N4-2 AstKO (B) mice before (Baseline) and after (Kainate) induction of $\gamma$-oscillations with $100 \mathrm{nM}$ kainate application. (C) Representative recordings in the CA3 region of acute hippocampal slices from N4-2 AstKO mice before (Baseline) and after (Kainate + Fluo) induction of $\gamma$-oscillations with $100 \mathrm{nM}$ kainate application in the presence of fluoxetine. (D and E) Average powers (D) and frequencies (E) of $\gamma$-oscillations in N4-2 CTL (black dots) and N4-2 AstKO without (green dots) and with (gray dots) fluoxetine. (F to $\mathbf{H})$ Representative recordings from the same set of experiments as (A) to (C), except for the usage of GAP26 as a pharmacological blocker in (H). (I and J) Average powers (I) and frequencies (J) of $\gamma$-oscillations in N4-2 CTL (black dots) and N4-2 AstKO hippocampal slices without (green dots) and with (gray dots) GAP26. Results are shown as mean \pm SEM. Numbers of recorded slices (n); (D) and (E), $n=33$ for N4-2 CTL, $n=32$ for N4-2 AstKO, $n=40$ for N4-2 AstKO + Fluo; (I) and (J), $n=19$ for N4-2 CTL, $\mathrm{n}=27$ for N4-2 AstKO, $\mathrm{n}=19$ for N4-2 AstKO + GAP26. ***, $p<0.001$; **, $0.001<p<0.01 ; *, 0.01<p<0.05$; no asterisk, $0.05<p$ (one-way ANOVA with Newman-Keuls test for $\mathbf{D}, \mathbf{E}, \mathbf{I}$, and $\mathbf{J})$. See also Tables S3 and S4. 
A

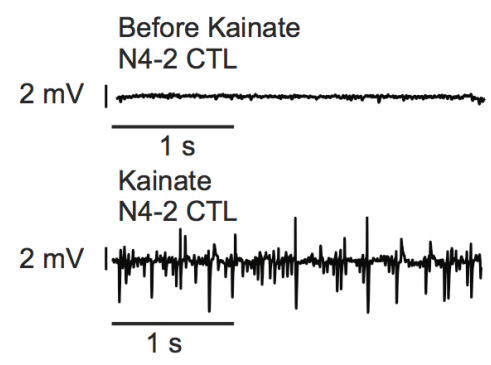

\section{Kainate}

$1 \mathrm{mV}$
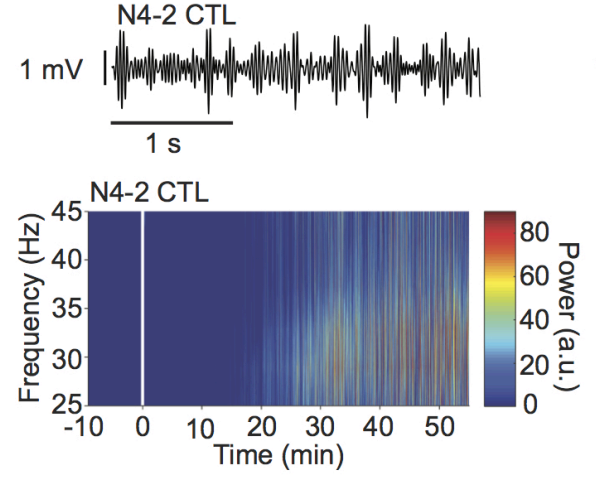

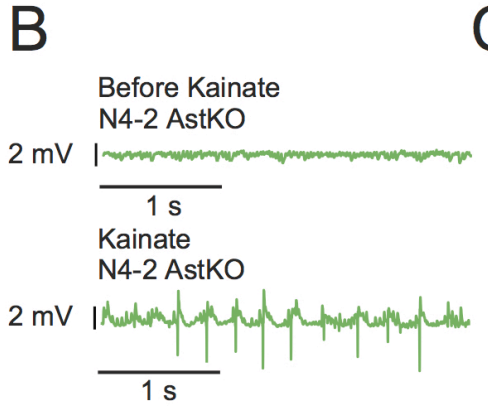

Kainate

N4-2 AstKO

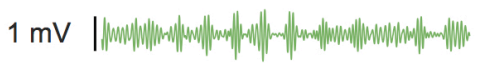

$1 \mathrm{~s}$

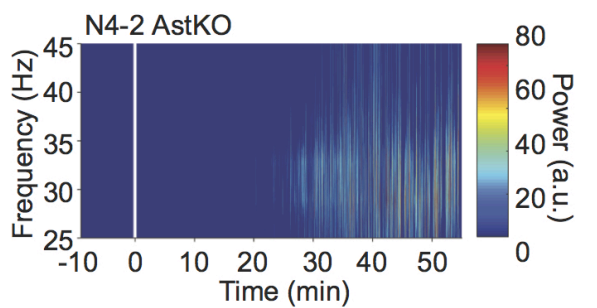

Figure 9. Decreased $\gamma$-oscillations in N4-2 AstKO mice in vivo. (A and B) Representative local field potentials (LFP) recorded in the CA3 region of the hippocampus in vivo from anesthetized N4-2 CTL (A) and from N4-2 AstKO (B) mice before (Before Kainate) and after (Kainate) intraperitoneal injection of kainate. Third traces in (A) and (B) are representative slow- $\gamma$ filtered LFPs. Bottom heat maps are representative normalized spectrograms. Kainate was injected at time point $0 \mathrm{~min}$. (C) The normalized average power of slow- $\gamma$-band oscillation in N4-2 CTL (black trace) and N4-2 AstKO (green trace) mice. Slow $\gamma$-band oscillation in N4-2 AstKO showed a siginficantly reduced power in comparison to the N4-2 CTL. Results are shown as mean \pm SEM. $\mathrm{N}=4$ animals for N4-2 CTL and $\mathrm{N}=4$ animals for N4-2 AstKO. ${ }^{*}, p<0.05$ (repeated measures two-way ANOVA with LSD posthoc test). See also Table S5. 


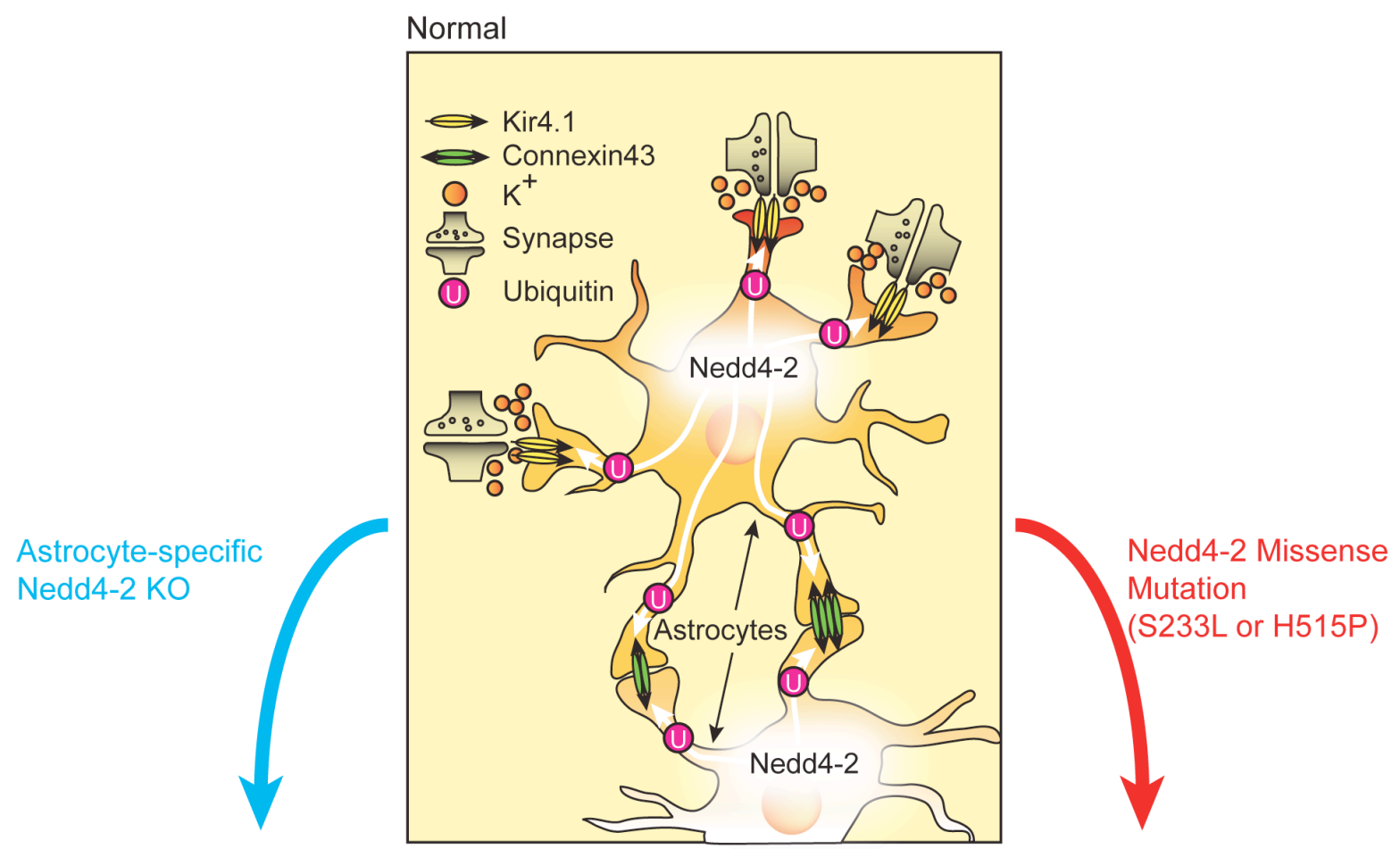

Loss-of-Function of Nedd4-2

(Reduced $\gamma$-Oscillation)

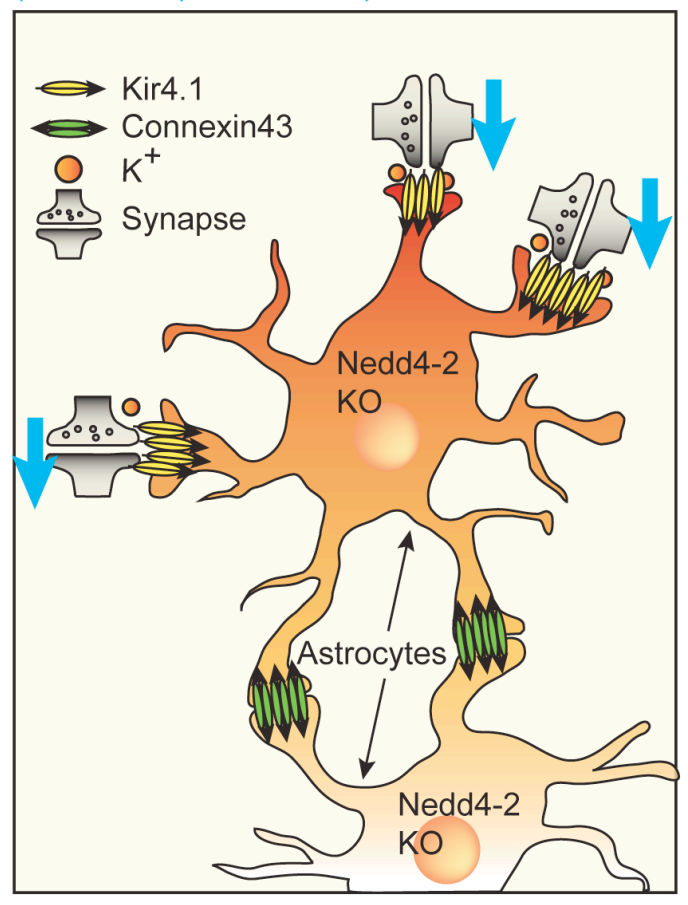

Gain-of-Function of Nedd4-2 (Epilepsy)

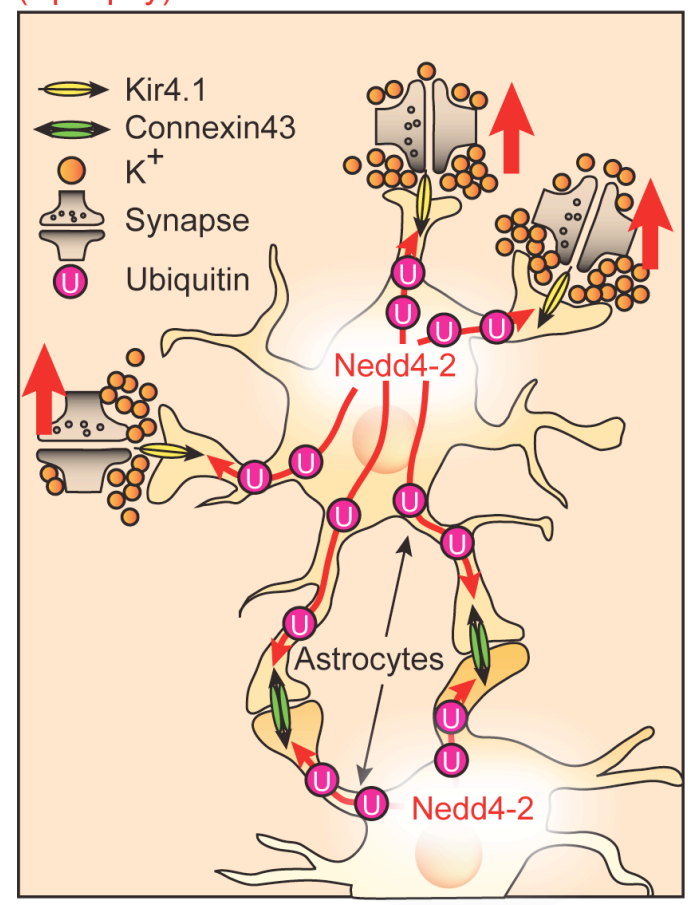

Figure 10. Physiological and pathophysiological homeostasis of astroglial channel proteins by Nedd4-2. (Top panel) Kir4.1 and Cx43 are ubiquitinated by Nedd4-2 and thus degraded by lysosome in astrocytes. This process limits the uptake of extracellular potassium and subsequent dissipation of intracellular potassium via gap junctions. A sustained extracellular potassium is critical for the maintenance of the neuronal network activity. (Left bottom panel) In astrocyte-specific Nedd4-2 KO mouse, the neuronal network activity is depressed because of reduced extracellular potassium caused by the augmentation of Kir4.1 and Cx43 levels in astrocytes. (Right bottom panel) Nedd4-2 missense mutation (S233L or H515P) causes the gain-of-function of E3 ligase activity, increasing in ubiquitination levels 
of Kir4.1 and Cx43, and thus reducing their protein expression. This is the potential cause of an enhanced neuronal netwotk activity and epilepsy. 